\title{
Combinatorics of open covers (VII): Groupability
}

\author{
by \\ Ljubiša D. R. Kočinac (Niš) and Marion Scheepers (Boise, ID)
}

\begin{abstract}
We use Ramseyan partition relations to characterize:
- the classical covering property of Hurewicz;

- the covering property of Gerlits and Nagy;

- the combinatorial cardinal numbers $\mathfrak{b}$ and $\operatorname{add}(\mathcal{M})$.
\end{abstract}

Let $X$ be a $\mathrm{T}_{3 \frac{1}{2}}$-space. In [9] we showed that $\mathrm{C}_{\mathrm{p}}(X)$ has countable strong fan tightness as well as the Reznichenko property if, and only if, all finite powers of $X$ have the GerlitsNagy covering property. Now we show that the following are equivalent:

1. $\mathrm{C}_{\mathrm{p}}(X)$ has countable fan tightness and the Reznichenko property.

2. All finite powers of $X$ have the Hurewicz property.

We show that for $C_{p}(X)$ the combination of countable fan tightness with the Reznichenko property is characterized by a Ramseyan partition relation. Extending the work in [9], we give an analogous Ramseyan characterization for the combination of countable strong fan tightness with the Reznichenko property on $\mathrm{C}_{\mathrm{p}}(X)$.

Introduction. We continue the investigation of combinatorial properties of open covers. The main theme in this paper, as in the previous ones, will be to show that certain concepts appearing in mathematical literature can be characterized by simple selection principles. These selection principles are then characterized game-theoretically and Ramsey-theoretically.

To ease the reader into our topic we recall the important basic definitions here in the introduction. Directly after that we give in Section 1 an abstract setting for the typical arguments used to derive Ramsey-theoretic results from game-theoretic circumstances. This, once and for all, will codify arguments that have repeatedly shown up in the study of selection princi-

2000 Mathematics Subject Classification: 54D20, 54C35, 54A25, 03E02, 91A44.

Key words and phrases: Hurewicz property, Reznichenko property, Gerlits-Nagy property $(*)$, groupability, $\omega$-cover, countable fan tightness, countable strong fan tightness, Ramsey theory, game theory, $\mathfrak{b}, \operatorname{add}(\mathcal{M})$.

Research of L. Kočinac supported by the Serbian Scientific Foundation, grant $\mathrm{N}^{0}$ 04M01.

Research of M. Scheepers supported by NSF grant DMS 99-71282. 
ples. In Section 2 we give an abstract treatment of one of the typical arguments for deriving a selection hypothesis from a Ramseyan partition relation. Though the setting developed here may not codify all circumstances under which selection hypotheses can be derived from Ramseyan hypotheses, it does cover the situations arising in the context of covering properties. In Section 3 we introduce the main combinatorial property featured in this paper - groupability. To be sure, this property is not new to us or the literature either. Only, we finally realized its significance in some classical notions. In Section 4 we give some examples to illustrate the concepts and ideas from the first three sections. Then in Section 5 we characterize the classical Hurewicz property (introduced in 1925) by a selection principle analogous to one that Hurewicz used to characterize a property that was introduced in 1924 by Menger. It is also characterized game-theoretically and Ramseytheoretically. The combinatorial cardinal $\mathfrak{b}$ is closely related to the Hurewicz property. We use the techniques developed here to give a new game-theoretic characterization, as well as a Ramsey-theoretic characterization of $\mathfrak{b}$. In Section 6 we characterize a covering property of Gerlits and Nagy (introduced in 1981) by a selection principle analogous to one introduced by Rothberger (in 1938) and we also give game-theoretic and Ramsey-theoretic characterizations. This property is closely related to $\operatorname{add}(\mathcal{M})$, the additivity of the ideal of first category subsets of the real line. We use the techniques from this section to give a new game-theoretic, as well as a Ramsey-theoretic, characterization of $\operatorname{add}(\mathcal{M})$.

Experience shows that certain covering properties of a Tikhonov space $X$ manifest themselves as closure properties of the corresponding space $C_{p}(X)$ of real-valued continuous functions on $X$, endowed with the topology of pointwise convergence. In Section 7 we connect the Hurewicz property on $X$ with Arkhangel'skiil's property of countable fan tightness, and a property of Reznichenko (from Section 3), on $C_{p}(X)$. In particular we prove the expected "duality" between $X$ and $\mathrm{C}_{\mathrm{p}}(X)$ for these properties, including a Ramseyan characterization of the closure property on $C_{p}(X)$. In Section 8 we connect the Gerlits-Nagy property (from Section 5) with Sakai's property of countable strong fan tightness, and a property of Reznichenko, on $\mathrm{C}_{\mathrm{p}}(X)$. Also here the expected duality is proven, and game-theoretic and Ramsey-theoretic characterizations are given.

And now for the promised basic definitions:

The selection hypothesis $\mathrm{S}_{1}(\mathcal{A}, \mathcal{B})$. Let $S$ be an infinite set and let $\mathcal{A}$ and $\mathcal{B}$ be collections of subsets of $S$. Then the symbol $\mathrm{S}_{1}(\mathcal{A}, \mathcal{B})$ denotes the statement:

For each sequence $\left(A_{n}: n<\infty\right)$ of elements of $\mathcal{A}$ there is a sequence $\left(b_{n}: n<\infty\right)$ such that for each $n$ we have $b_{n} \in A_{n}$, and $\left\{b_{n}: n<\infty\right\} \in \mathcal{B}$. 
Here are some examples from the literature. Let $\mathcal{O}$ denote the collection of all open covers of $X$. Rothberger introduced the property $\mathrm{S}_{1}(\mathcal{O}, \mathcal{O})$ in [17].

According to [5] a cover $\mathcal{U}$ of $X$ is an $\omega$-cover if: $X$ is not a member of $\mathcal{U}$, each element of $\mathcal{U}$ is an open set, and for each finite subset $F$ of $X$ there is a $U \in \mathcal{U}$ such that $F \subset U$. We shall use the symbol $\Omega$ to denote the collection of $\omega$-covers of $X$. Sakai introduced the property $\mathrm{S}_{1}(\Omega, \Omega)$ in [18] and showed that a space has property $\mathrm{S}_{1}(\Omega, \Omega)$ if, and only if, it has property $\mathrm{S}_{1}(\mathcal{O}, \mathcal{O})$ in all finite powers. Throughout we shall assume that each $\omega$-cover of the space on which we consider it, has a countable subset which is an $\omega$-cover. According to [5] spaces with this property are called $\epsilon$-spaces. It was shown in [5] that these are the spaces which have the Lindelöf property in all their finite powers.

For $X$ a space and for $x \in X$ the symbol $\Omega_{x}$ denotes the set $\{A \subset$ $X \backslash\{x\}: x \in \bar{A}\}$. A space is said to have countable tightness at $x$ if each element of $\Omega_{x}$ has a countable subset which is in $\Omega_{x}$. According to [18], $X$ has countable strong fan tightness at $x$ if $\mathrm{S}_{1}\left(\Omega_{x}, \Omega_{x}\right)$ holds.

In Section 5 we show that a property of Gerlits and Nagy is characterized by a hypothesis of the form $\mathrm{S}_{1}(\mathcal{A}, \mathcal{B})$. In Section 7 we show how to characterize a corresponding property on $\mathrm{C}_{\mathrm{p}}(X)$ by this selection hypothesis.

The selection hypothesis $\mathrm{S}_{\mathrm{fin}}(\mathcal{A}, \mathcal{B})$. The symbol $\mathrm{S}_{\mathrm{fin}}(\mathcal{A}, \mathcal{B})$ denotes:

For each sequence $\left(A_{n}: n<\infty\right)$ of elements of $\mathcal{A}$ there is a sequence $\left(B_{n}: n<\infty\right)$ of finite sets such that for each $n$ we have $B_{n} \subset A_{n}$, and $\bigcup_{n<\infty} B_{n} \in \mathcal{B}$.

Here are examples from the literature: Hurewicz showed in [6] that a property introduced by Menger in [12] is equivalent to the property $\mathrm{S}_{\text {fin }}(\mathcal{O}, \mathcal{O})$. To distinguish it from another covering property introduced by Hurewicz, this is often called the Menger property. In [8] it was shown that a space has property $\mathrm{S}_{\text {fin }}(\Omega, \Omega)$ if, and only if, it has property $\mathrm{S}_{\text {fin }}(\mathcal{O}, \mathcal{O})$ in all finite powers.

According to [1] a space has countable fan tightness at $x$ if $\mathrm{S}_{\text {fin }}\left(\Omega_{x}, \Omega_{x}\right)$ holds.

In Section 4 we characterize the Hurewicz property by a hypothesis of the form $\mathrm{S}_{\mathrm{fin}}(\mathcal{A}, \mathcal{B})$, and we characterize in Section 6 the corresponding property on $\mathrm{C}_{\mathrm{p}}(X)$ by a selection hypothesis of this form.

Corresponding infinite games. Sometimes the two selection properties introduced above are characterized by games. The symbol $\mathrm{G}_{1}(\mathcal{A}, \mathcal{B})$ denotes the game where two players, ONE and TWO, play an inning per positive integer. In the $n$th inning ONE chooses a set $O_{n}$ from $\mathcal{A}$, and TWO responds by choosing an element $T_{n} \in O_{n}$. The play $\left(O_{1}, T_{1}, \ldots, O_{n}, T_{n}, \ldots\right)$ is won 
by TWO if $\left\{T_{n}: n<\infty\right\}$ is a member of $\mathcal{B}$; otherwise, ONE wins. The earliest published example of a game of this form appeared in [4], where Galvin introduced the game $\mathrm{G}_{1}(\mathcal{O}, \mathcal{O})$. Pawlikowski proved in [14] that a space has property $\mathrm{S}_{1}(\mathcal{O}, \mathcal{O})$ if, and only if, ONE has no winning strategy in the game $\mathrm{G}_{1}(\mathcal{O}, \mathcal{O})$.

The symbol $\mathrm{G}_{\text {fin }}(\mathcal{A}, \mathcal{B})$ denotes the game where ONE and TWO play an inning per positive integer, and in the $n$th inning ONE chooses a set $O_{n} \in \mathcal{A}$, while TWO responds by choosing a finite set $T_{n} \subseteq O_{n}$. The play $\left(O_{1}, T_{1}, \ldots, O_{n}, T_{n}, \ldots\right)$ is won by TWO if $\bigcup_{n<\infty} T_{n} \in \mathcal{B}$; otherwise, ONE wins. Though a game of this form was first explicitly defined by Telgársky in [25], it was already considered in 1925 by Hurewicz when he proved in Theorem 10 of $[6]$ that a space has property $\operatorname{S}_{\text {fin }}(\mathcal{O}, \mathcal{O})$ if, and only if, ONE has no winning strategy in the game $\mathrm{G}_{\text {fin }}(\mathcal{O}, \mathcal{O})$.

It is evident that if ONE does not have a winning strategy in the game $\mathrm{G}_{1}(\mathcal{A}, \mathcal{B})$ then the selection hypothesis $\mathrm{S}_{1}(\mathcal{A}, \mathcal{B})$ is true. Similar remarks apply to $\mathrm{G}_{\text {fin }}(\mathcal{A}, \mathcal{B})$ and $\mathrm{S}_{\text {fin }}(\mathcal{A}, \mathcal{B})$. It is the converse implication - the selection hypothesis implies that ONE has no winning strategy in the corresponding game - which is not always true. When the converse implication is true, the game characterizes the selection principle, and is a powerful tool to extract additional information about $\mathcal{A}$ and $\mathcal{B}$. If $\mathcal{A}$ has appropriate properties, the game-theoretic characterization can be used to derive Ramsey-theoretic statements.

Ramsey-theoretic statements. For positive integers $n$ and $k$ the symbol

$$
\mathcal{A} \rightarrow(\mathcal{B})_{k}^{n}
$$

denotes the following statement:

For each $A \in \mathcal{A}$ and for each function $f:[A]^{n} \rightarrow\{1, \ldots, k\}$ there are a set $B \subseteq A$ and a $j \in\{1, \ldots, k\}$ such that for each $Y \in[B]^{n}$, $f(Y)=j$, and $B \in \mathcal{B}$.

Here, as elsewhere, the symbol $[A]^{n}$ denotes the set of $n$-element subsets of $A$. It is customary to call $f$ a "coloring", and to say that " $B$ is homogeneous of color $j$ for $f$ ".

This symbol is called the ordinary partition symbol and has a vast literature. It is one of several such partition symbols that have been extensively studied. The area of mathematics dealing with partition symbols is called Ramsey Theory after F. P. Ramsey who proved the first important partition theorem. Ramsey's famous theorem in [16] states: Let $\mathcal{A}$ be the collection of infinite subsets of the integers. Then for each $n$ and each $k, \mathcal{A} \rightarrow(\mathcal{A})_{k}^{n}$. The ordinary partition symbol denotes a relation between $\mathcal{A}$ and $\mathcal{B}$, and this relation is customarily called the ordinary partition relation. Several selection principles of the form $\mathrm{S}_{1}(\mathcal{A}, \mathcal{B})$ have been characterized by the ordinary par- 
tition relation. In [24] it was, for example, shown that a space has property $\mathrm{S}_{1}(\mathcal{O}, \mathcal{O})$ if, and only if, for all $k$ one has $\Omega \rightarrow(\mathcal{O})_{k}^{2}$.

Another partition symbol that will be important for us comes from a study of Baumgartner and Taylor in [3]. To be precise, the notion is defined there, but the notation arose elsewhere. For each positive integer $k$,

$$
\mathcal{A} \rightarrow\lceil\mathcal{B}\rceil_{k}^{2}
$$

denotes the following statement:

For each $A$ in $\mathcal{A}$ and for each function $f:[A]^{2} \rightarrow\{1, \ldots, k\}$ there is a set $B \subset A$ and a $j \in\{1, \ldots, k\}$, and a partition $B=\bigcup_{n<\infty} B_{n}$ of $B$ into pairwise disjoint finite sets such that for each $\{a, b\} \in[B]^{2}$ for which $a$ and $b$ are not from the same $B_{n}$, we have $f(\{a, b\})=j$, and $B \in \mathcal{B}$.

It is customary to say that " $B$ is nearly homogeneous for $f$ ". The relation between $\mathcal{A}$ and $\mathcal{B}$ denoted by this partition symbol will be called the Baumgartner-Taylor partition relation. Several selection principles of the form $\mathrm{S}_{\text {fin }}(\mathcal{A}, \mathcal{B})$ have been characterized by the Baumgartner-Taylor partition relation. For example, in [24] it was shown that a space has property $\mathrm{S}_{\mathrm{fin}}(\mathcal{O}, \mathcal{O})$ if, and only if, for each $k$ one has $\Omega \rightarrow\lceil\mathcal{O}\rceil_{k}^{2}$.

1. From games to partition relations. Call a family $\mathcal{A}$ of sets persistent if: (i) No finite set is a member of $\mathcal{A}$ and (ii) for each $A \in \mathcal{A}$ and for each partition of $A$ into finitely many sets, at least one of these sets is again a member of $\mathcal{A}$.

The second requirement for persistence could be stated in the form: For each $k, \mathcal{A} \rightarrow(\mathcal{A})_{k}^{1}$.

For instance, $\Omega$ is a persistent family.

THEOREM 1. If $\mathcal{A}$ is a persistent family of countable subsets of a set $S$, and if $O N E$ has no winning strategy in the game $\mathrm{G}_{1}(\mathcal{A}, \mathcal{B})$ played on $S$, then for each $k$ the ordinary partition relation $\mathcal{A} \rightarrow(\mathcal{B})_{k}^{2}$ holds.

Proof. Let $A \in \mathcal{A}$ as well as a positive integer $k$ be given. Enumerate $A$ bijectively as $\left(a_{n}: n<\infty\right)$. Fix a function $f:[A]^{2} \rightarrow\{1, \ldots, k\}$. Recursively define a sequence $\left(A_{n}: n<\infty\right)$ of subsets of $A$, and a sequence $\left(i_{n}: n<\infty\right)$ of elements of $\{1, \ldots, k\}$ so that:

1. For each $n, A_{n} \in \mathcal{A}$ and $A_{n+1} \subset A_{n}$.

2. For each $n, A_{n+1}=\left\{a_{j} \in A_{n}: j>n+1\right.$ and $\left.f\left(\left\{a_{n+1}, a_{j}\right\}\right)=i_{n+1}\right\}$.

To see that this can be done, first observe that putting $B_{j}=\left\{a_{i} \in A\right.$ : $i>1$ and $\left.f\left(\left\{a_{1}, a_{i}\right\}\right)=j\right\}$ we get a partition of $A \backslash\left\{a_{1}\right\}$ into finitely many pieces. Since $\mathcal{A}$ is persistent there is a $j$ for which $B_{j}$ is in $\mathcal{A}$. Fix such a $j$ and set $i_{1}=j, A_{1}=B_{j}$. Next observe that by similar argument the 
remaining terms of an infinite sequence as above can be selected consecutively.

Next, for each $j \in\{1, \ldots, k\}$ define $E_{j}=\left\{a_{n}: i_{n}=j\right\}$. For each $n$, $A_{n} \cap E_{1}, \ldots, A_{n} \cap E_{k}$ partitions $A_{n}$ into finitely many pieces, and so there is a $j_{n}$ with $A_{n} \cap E_{j_{n}}$ in $\mathcal{A}$. Since for each $n$ we have $A_{n} \supset A_{n+1}$, we may assume that the same $j$ works for all $A_{n}$ 's. Fix such a $j$.

Now define the following strategy, $\sigma$, for ONE in the game $\mathrm{G}_{1}(\mathcal{A}, \mathcal{B})$ : In the first inning, $\sigma(\emptyset)=A_{1} \cap E_{j}$. If TWO responds by choosing $a_{n_{1}} \in \sigma(\emptyset)$, then ONE plays $\sigma\left(a_{n_{1}}\right)=A_{n_{1}} \cap E_{j}$. If TWO responds with $a_{n_{2}} \in \sigma\left(a_{n_{1}}\right)$, then ONE plays $\sigma\left(a_{n_{1}}, a_{n_{2}}\right)=A_{n_{2}} \cap E_{j}$, and so on. (Observe that $n_{1}<n_{2}$.)

Since ONE has no winning strategy in $\mathrm{G}_{1}(\mathcal{A}, \mathcal{B})$, there is a play during which ONE used $\sigma$ but lost. Let

$$
\sigma(\emptyset), a_{n_{1}}, \sigma\left(a_{n_{1}}\right), a_{n_{2}}, \sigma\left(a_{n_{1}}, a_{n_{2}}\right), a_{n_{3}}, \ldots
$$

be such a play lost by ONE. Then as TWO won we have $B=\left\{a_{n_{i}}: i<\infty\right\}$ $\in \mathcal{B}$. By the definition of the strategy $\sigma$ we also find for $r \neq s$ that $n_{r} \neq n_{s}$, and $f\left(\left\{a_{n_{r}}, a_{n_{s}}\right\}\right)=j$, and so $B$ is homogeneous of color $j$ for $f$.

COROLlary 2. If $\mathcal{A}$ is a persistent family of countable sets and if ONE has no winning strategy in the game $\mathrm{G}_{1}(\mathcal{A}, \mathcal{A})$, then for each $n$ and $k$ the ordinary partition relation $\mathcal{A} \rightarrow(\mathcal{A})_{k}^{n}$ holds.

Proof. By Theorem 1 we see that for each $k, \mathcal{A} \rightarrow(\mathcal{A})_{k}^{2}$. Starting from this case of the ordinary partition relation and the fact that $\mathcal{A}$ is persistent one then uses a standard induction (see e.g. the proof of Theorem A of [16]) on $n$ and $k$ to prove $\mathcal{A} \rightarrow(\mathcal{A})_{k}^{n}$.

TheOREM 3. If $\mathcal{A}$ is a persistent family and if ONE has no winning strategy in the game $\mathrm{G}_{\mathrm{fin}}(\mathcal{A}, \mathcal{B})$, then for each $k$ the Baumgartner-Taylor partition relation $\mathcal{A} \rightarrow\lceil\mathcal{B}\rceil_{k}^{2}$ holds.

Proof. Let $A \in \mathcal{A}$ and $f:[A]^{2} \rightarrow\{1, \ldots, k\}$ be given. Proceed as in the proof of Theorem 1 to construct the $A_{n}$, and choose a $j$ and $E_{j}$ exactly as there. Then change the argument as follows at the stage where a strategy for ONE is defined:

Define a strategy $\sigma$ for ONE as follows: $\sigma(\emptyset)=A_{1} \cap E_{j}$. If TWO responds with the finite set $T_{1} \subset \sigma(\emptyset)$, then one puts $n_{1}=\max \left\{n: a_{n} \in T_{1}\right\}$, and plays $\sigma\left(T_{1}\right)=A_{n_{1}} \cap E_{j}$. Should TWO now respond with the finite set $T_{2} \subset \sigma\left(T_{1}\right)$, then ONE computes $n_{2}=\max \left\{n: a_{n} \in T_{2}\right\}$ and plays $\sigma\left(T_{1}, T_{2}\right)=A_{n_{2}} \cap E_{j}$, and so on. Observe that by the definition of the $A_{j}$ 's we will have $n_{1}<n_{2}<\ldots$, and $T_{r} \cap T_{s}=\emptyset$ whenever $r \neq s$, during any $\sigma$-play of the game.

Then invoke the fact that ONE has no winning strategy in $\mathrm{G}_{\text {fin }}(\mathcal{A}, \mathcal{B})$ to obtain a $\sigma$-play

$$
\sigma(\emptyset), T_{1}, \sigma\left(T_{1}\right), T_{2}, \sigma\left(T_{1}, T_{2}\right), T_{3}, \ldots
$$


lost by ONE. One then checks that TWO's moves produce the required nearly homogeneous set for $f$ in $\mathcal{B}$.

2. From partition relations to selection hypotheses. We now give general arguments for deriving from a partition relation a selection hypothesis of the form $\mathrm{S}_{\text {fin }}(\mathcal{A}, \mathcal{B})$ or of the form $\mathrm{S}_{1}(\mathcal{A}, \mathcal{B})$. Let $S$ be an infinite set. The pair $(\mathcal{A}, \mathcal{B})$ is selectable if:

S1 $\mathcal{A}$ and $\mathcal{B}$ are subsets of $\mathcal{P}(\mathcal{P}(S))$.

S2 For each countably infinite $A \in \mathcal{A}$ and for each sequence $\left(A_{n}: n<\infty\right)$ from $\mathcal{A}$ and for each 1-to-1 onto function $f: A \rightarrow \mathbb{N}$ the set

$$
\left\{U \cap V: U \in A, V \in A_{f(U)}\right\} \backslash\{\emptyset\}
$$

is an element of $\mathcal{A}$.

S3 Each element of $\mathcal{A}$ has a countable subset which is an element of $\mathcal{A}$.

S4 For each $A \in \mathcal{A}$ and for each $X \in A, \mathcal{P}(\mathcal{P}(X)) \cap \mathcal{B}=\emptyset$.

S5 For each $B \in \mathcal{B}$, for each finite $F \subset B$, also $B \backslash F$ is in $\mathcal{B}$.

S6 If $C$ is a countable set of subsets of $S$ which has the properties:

(1) there is a $B$ in $\mathcal{B}$ such that $\{b \in B:(\exists X \in C)(b \subseteq X)\} \in \mathcal{B}$;

(2) for each $X \in C$ there is an $A \in \mathcal{A}$ with $X \in A$;

then $C$ is a member of $\mathcal{B}$.

Observe that if $C$ satisfies (1) and (2) of S6, then by S5 for each finite $F \subset C$, also $C \backslash F$ satisfies S6(1), (2).

Theorem 4. If $(\mathcal{A}, \mathcal{B})$ is a selectable pair and $\mathcal{A} \rightarrow\lceil\mathcal{B}\rceil_{2}^{2}$, then $\mathrm{S}_{\mathrm{fin}}(\mathcal{A}, \mathcal{B})$ holds.

Proof. Let $\left(\mathcal{U}_{n}: n<\infty\right)$ be a sequence of elements of $\mathcal{A}$. By S3 we may assume each $\mathcal{U}_{n}$ is countable, and enumerate it as $\left(U_{k}^{n}: k<\infty\right)$. Define $\mathcal{V}$ to be the collection of nonempty sets of the form $U_{n}^{1} \cap U_{k}^{n}$. By $\mathrm{S} 2, \mathcal{V}$ is an element of $\mathcal{A}$. Choose for each element of $\mathcal{V}$ a representation of the form $U_{n}^{1} \cap U_{k}^{n}$.

Define a function $f:[\mathcal{V}]^{2} \rightarrow\{1,2\}$ by

$$
f\left(\left\{U_{n_{1}}^{1} \cap U_{k}^{n_{1}}, U_{n_{2}}^{1} \cap U_{j}^{n_{2}}\right\}\right)= \begin{cases}1 & \text { if } n_{1}=n_{2}, \\ 2 & \text { otherwise. }\end{cases}
$$

Choose a $\mathcal{W} \subseteq \mathcal{V}$ nearly homogeneous of color $j$ with $\mathcal{W} \in \mathcal{B}$. Let $\left(\mathcal{W}_{k}\right.$ : $k<\infty$ ) be a sequence of finite sets, disjoint from each other and with union $\mathcal{W}$, such that for $A$ and $B$ from distinct $\mathcal{W}_{k}$ 's, $f(\{A, B\})=j$.

CASE 1: $j=1$. Then there is an $n$ such that for all $A \in \mathcal{W}$ we have $A \subset U_{n}^{1}$. Then S5 implies that $\mathcal{W}$ is not an element of $\mathcal{B}$. Thus, Case 1 does not hold. 
CAsE 2: $j=2$. For each $k>1$ define $\mathcal{G}_{k}$ to be the set of $U_{j}^{k}$ which occur as second coordinate in the chosen representations of elements of $\mathcal{W}$. Then each $\mathcal{G}_{k}$ is a finite subset of $\mathcal{U}_{k}$. Put $\mathcal{G}=\bigcup_{k<\infty} \mathcal{G}_{k}$.

Then $\mathcal{W}=\{b \in \mathcal{W}:(\exists x \in \mathcal{G})(b \subseteq x)\}$ is in $\mathcal{B}$, and each element of $\mathcal{G}$ is an element of some member of $\mathcal{A}$. Thus by $\mathrm{S} 6, \mathcal{G}$ is an element of $\mathcal{B}$. By letting $\mathcal{G}_{k}$ be empty for those $k$ for which no $\mathcal{G}_{k}$ was defined, we find that the sequence $\left(\mathcal{G}_{k}: k<\infty\right)$ witnesses $\operatorname{S}_{\text {fin }}(\mathcal{A}, \mathcal{B})$ for $\left(\mathcal{U}_{k}: k<\infty\right)$.

THEOREM 5. If $(\mathcal{A}, \mathcal{B})$ is a selectable pair and $\mathcal{A} \rightarrow(\mathcal{B})_{2}^{2}$ holds, then $\mathrm{S}_{1}(\mathcal{A}, \mathcal{B})$ holds.

Proof. Let $\left(\mathcal{U}_{n}: n<\infty\right)$ be a sequence of elements of $\mathcal{A}$. Argue as above up to the definition of the coloring $f$.

Then choose a set $\mathcal{W} \subseteq \mathcal{V}$ homogeneous of color $j$ with $\mathcal{W} \in \mathcal{B}$. As in the preceding proof we conclude that $j=2$. Then for each $k$ for which this is possible, choose $U_{n_{k}}^{k}$ to be the second term in the chosen representation of a member of $\mathcal{W}$. For each $k$ such that none of the chosen sets is from $\mathcal{U}_{k}$ choose a $U_{n_{k}}^{k} \in \mathcal{U}_{k}$ arbitrarily. Let $\mathcal{G}$ be the set of $U_{n_{k}}^{k}$ 's. Then by $\mathrm{S} 6, \mathcal{G}$ is in $\mathcal{B}$, and it witnesses $\mathrm{S}_{1}(\mathcal{A}, \mathcal{B})$ for the sequence of $\mathcal{U}_{k}$ 's.

3. Groupability and countable distinct representatives. Let a family $\mathcal{A}$ of subsets of the infinite set $S$ be given. An element $A$ of $\mathcal{A}$ is said to be groupable if there is a partition $A=\bigcup_{n<\infty} A_{n}$ of $A$ into pairwise disjoint finite sets such that for each infinite set $N$ of positive integers, also $\bigcup\left\{A_{n}: n \in N\right\}$ is in $\mathcal{A}$. We shall use the notation $\mathcal{A}^{\text {gp }}$ to denote the set of groupable elements of $\mathcal{A}$.

LEMma 6. (1) An $\omega$-cover $\mathcal{U}$ of a space $X$ is groupable if there is a partition $\left(\mathcal{U}_{n}: n<\infty\right)$ of $\mathcal{U}$ into pairwise disjoint finite sets such that: For each finite subset $F$ of $X$, for all but finitely many $n$, there is a $U \in \mathcal{U}_{n}$ such that $F$ is a subset of $U$.

(2) An element $A$ of $\Omega_{x}$ is groupable if there is a partition $\left(A_{n}: n<\infty\right)$ of $A$ into pairwise disjoint finite sets such that each neighborhood of $x$ has nonempty intersection with all but finitely many of the $A_{n}$.

(3) An open cover $\mathcal{U}$ is groupable if, and only if, there is a partition $\mathcal{U}=\bigcup_{n<\infty} \mathcal{U}_{n}$ where the $\mathcal{U}_{n}$ 's are finite and disjoint from each other, such that each point in the space belongs to all but finitely many of the sets $\cup \mathcal{U}_{n}$.

Proof. Exercise.

In 1996 Reznichenko introduced (in a seminar at Moscow State University) the following property: Each countable element of $\Omega_{x}$ is a member of $\Omega_{x}^{\mathrm{gp}}$. The property was further studied in [9] and in [11]. In [9] we called this the Reznichenko property at $x$. When $X$ has the Reznichenko property at each of its points, then $X$ is said to have the Reznichenko property. In 
[6] Hurewicz introduced the following covering property on a space $X$ (it is nowadays called the Hurewicz property): For each sequence $\left(\mathcal{U}_{n}: n<\infty\right)$ of open covers there is a sequence $\left(\mathcal{V}_{n}: n<\infty\right)$ such that each $\mathcal{V}_{n}$ is a finite subset of $\mathcal{U}_{n}$, and each element of the space belongs to all but finitely many of the sets $\cup \mathcal{V}_{n}$. The Hurewicz game on a space $X$ is played as follows. Players ONE and TWO play an inning per positive integer. In the $n$th inning ONE chooses an open cover $O_{n}$ of $X$, and TWO responds by choosing a finite set $T_{n} \subset O_{n}$. A play $\left(O_{1}, T_{1}, \ldots, O_{n}, T_{n}, \ldots\right)$ is won by TWO if for each $x \in X$ the set $\left\{n: x \notin \cup T_{n}\right\}$ is finite. By [19, Theorem 27], $X$ has the Hurewicz property if, and only if, ONE has no winning strategy in the Hurewicz game.

An open cover of a space is said to be large if there are for each point infinitely many sets in the cover containing that point. The symbol $\Lambda$ will be used to denote the collection of large covers of a space.

Lemma 7. If $X$ has the Hurewicz property then each countable large cover of $X$ is groupable-i.e., each countable element of $\Lambda$ is in $\Lambda^{\text {gp }}$.

Proof. Let $\mathcal{U}$ be a (countable) large open cover of $X$. Consider the following strategy, $\sigma$, of ONE in the Hurewicz game on $X$. In the first inning ONE plays $\sigma(\emptyset)=\mathcal{U}$. If TWO responds with the finite set $T_{1}$, then ONE plays $\sigma\left(T_{1}\right)=\mathcal{U} \backslash T_{1}$. If TWO responds with the finite set $T_{2} \subset \sigma\left(T_{1}\right)$, then ONE plays $\sigma\left(T_{1}, T_{2}\right)=\mathcal{U} \backslash\left(T_{1} \cup T_{2}\right)$, and so on.

Since $\sigma$ is not a winning strategy for ONE, consider a play

$$
\sigma(\emptyset), T_{1}, \sigma\left(T_{1}\right), T_{2}, \sigma\left(T_{1}, T_{2}\right), T_{3}, \ldots
$$

which is lost by ONE. Then the sequence $\left(T_{n}: n<\infty\right)$ of moves by TWO are disjoint from each other (by the definition of the strategy $\sigma$ ) and each element of $X$ is in all but finitely many of the sets $\cup T_{n}$. Thus, TWO's moves witness the groupability of the large cover $\bigcup_{n<\infty} T_{n}$ of $X$. If any elements of $\mathcal{U}$ are not present in the sequence of moves by TWO, then they can after the construction of the play be distributed among TWO's moves so that the result witnesses the groupability of $\mathcal{U}$.

In Lemma 5 of [9] we proved:

Lemma 8. If each finite power of $X$ has the Hurewicz property, then each $\omega$-cover of $X$ contains a groupable $\omega$-cover of $X-$ i.e., each countable element of $\Omega$ is in $\Omega^{\mathrm{gp}}$.

The combinatorial property $\operatorname{CDR}_{\text {Sub }}(\mathcal{A}, \mathcal{B})$ was introduced in [19]: For each sequence $\left(A_{n}: n<\infty\right)$ of elements of $\mathcal{A}$ there is a sequence $\left(B_{n}: n<\infty\right)$ of pairwise disjoint elements of $\mathcal{B}$ such that for each $n, B_{n} \subseteq A_{n}$. We say $\mathcal{A}$ is countably distinctly representable by $\mathcal{B}$. At first glance this property may appear somewhat unmotivated, but it is very useful in combinatorial 
arguments to follow. It is the "structure" counterpart of the well known fact that given a countable collection of infinite sets, one can find inside each of these sets a new infinite set such that the new sets are pairwise disjoint.

LEMma 9. For each family $\mathcal{A}$ of subsets of the infinite set $S$ the statement $\mathrm{CDR}_{\mathrm{Sub}}\left(\mathcal{A}^{\mathrm{gp}}, \mathcal{A}^{\mathrm{gp}}\right)$ holds.

Proof. We may assume that $\mathcal{A}^{\mathrm{gp}}$ is nonempty. Let $\left(A_{n}: n<\infty\right)$ be a sequence of elements of $\mathcal{A}^{\mathrm{gp}}$. For each $n$ choose a partition $\left(A_{m}^{n}: m<\infty\right)$ of $A_{n}$ into pairwise disjoint finite sets such that the chosen partition witnesses groupability of $A_{n}$. We must for each $n$ choose a subset $B_{n}$ of $A_{n}$ such that any two $B_{n}$ 's are disjoint from each other, and each $B_{n}$ is in $\mathcal{A}^{\mathrm{gp}}$.

This is done by a standard zig-zag argument: We define for each $n$ a function $f_{n}: \mathbb{N} \rightarrow \mathbb{N}$ as follows: To start, put $f_{1}(1)=1$ and $f_{1}(2)=2$. Then let $f_{2}(1)$ be the least $n$ such that for all $j \geq n, A_{j}^{2}$ is disjoint from the sets chosen so far- $A_{f_{1}(1)}^{1}$ and $A_{f_{1}(2)}^{1}$. Then let $f_{1}(3)$ be the least $n>f_{1}(2)$ such that for all $j \geq n, A_{j}^{1}$ is disjoint from all sets chosen so far. Then let $f_{2}(2)>f_{2}(1)$ be the least $n$ such that for all $j \geq n, A_{j}^{2}$ is disjoint from all sets chosen so far. Then let $f_{3}(1)$ be the least $n$ such that for all $j \geq n$, $A_{j}^{3}$ is disjoint from all sets chosen so far. Next choose $f_{1}(4)$ to be the least $n>f_{1}(3)$ such that for all $j \geq n, A_{j}^{1}$ is disjoint from all sets chosen so far. Then choose $f_{2}(3)$ to be the least $n>f_{2}(2)$ such that for all $j \geq n$, $A_{j}^{2}$ is disjoint from all sets chosen so far. Then choose $f_{3}(2)$ to be the least $n>f_{3}(1)$ so that for all $j \geq n, A_{j}^{3}$ is disjoint from all sets chosen so far. Choose $f_{4}(1)$ to be the least $n$ such that for all $j \geq n, A_{j}^{4}$ is disjoint from all sets chosen so far, and so on.

For each $n$ define $B_{n}=\bigcup_{j<\infty} A_{f_{n}(j)}^{n}$. For each $n$ the sequence $\left(A_{f_{n}(j)}^{n}\right.$ : $j<\infty)$ witnesses that $B_{n}$ is in $\mathcal{A}^{\mathrm{gp}}$. By construction any two $B_{n}$ 's are disjoint.

Corollary 10. (1) If $X$ has the Reznichenko property at $x$, then $\mathrm{CDR}_{\text {sub }}\left(\Omega_{x}, \Omega_{x}\right)$ holds.

(2) If $X$ has the Hurewicz property, then $\operatorname{CDR}_{\text {sub }}(\Lambda, \Lambda)$ holds.

(3) If each finite power of $X$ has the Hurewicz property, then $\operatorname{CDR}_{\text {sub }}(\Omega, \Omega)$ holds.

Now groupability is not the only property implying countable distinct representability. In Proposition 21 of [10] it was shown that $\mathrm{S}_{1}(\Omega, \Omega)$ implies $\operatorname{CDR}_{\text {sub }}(\Omega, \Omega)$; as we shall see, this could happen in the absence of groupability. In Theorem 16 of [19] it is shown that $S_{1}(\mathcal{O}, \mathcal{O})$ implies $\operatorname{CDR}_{\text {sub }}(\Lambda, \Lambda)$.

4. Illustrative examples. On request of a referee we include this section. The purpose is to give illustrations and examples of the preceding somewhat abstract treatment of how to derive Ramseyan statements from 
game-theoretic statements, and selection principles from Ramseyan statements. We will not quote all examples known to us, but merely point to a reasonable selection of examples.

For a space $X$ define $\mathcal{D}=\{\mathcal{U}:(\forall U \in \mathcal{U})(U$ open) and $\cup \mathcal{U}$ is dense in $X$ \}. We let $\mathcal{D}_{\Omega}$ denote the family of $\mathcal{U} \in \mathcal{D}$ such that $D \notin \mathcal{U}$ whenever $D$ is a dense open subset of $X$, and for each finite family $\mathcal{F}$ of open sets there is a $U \in \mathcal{U}$ such that for each $F \in \mathcal{F}$ we have $F \cap U \neq \emptyset$.

\section{From games to partition relations}

\section{Lemma 11. The families $\Omega, \Omega_{x}$ and $\mathcal{D}_{\Omega}$ are persistent.}

Thus if for a family $\mathcal{B}$ of open sets ONE has no winning strategy in the game $\mathrm{G}_{1}(\Omega, \mathcal{B})$, then by Theorem 1 the partition relation $\Omega \rightarrow(\mathcal{B})_{k}^{2}$ holds for each finite $k$. Now the implication $1 \Rightarrow 2$ of Theorem 4 of [24] states that for appropriate spaces $X$ : If $X$ satisfies $\mathrm{S}_{1}(\mathcal{O}, \mathcal{O})$ then for each positive integer $k, \Omega \rightarrow(\Lambda)_{k}^{2}$. Theorem 1 gives this implication as follows: A result of Pawlikowski in [14] implies that if $X$ has $\mathrm{S}_{1}(\mathcal{O}, \mathcal{O})$, then ONE has no winning strategy in $\mathrm{G}_{1}(\Omega, \Lambda)$. Then Theorem 1 gives the rest.

As a second example: If in a space of countable tightness ONE has no winning strategy in the game $\mathrm{G}_{1}\left(\Omega_{x}, \Omega_{x}\right)$, then by Corollary 2 the partition relation $\Omega_{x} \rightarrow\left(\Omega_{x}\right)_{k}^{n}$ holds for all finite $n$ and $k$. This is the implication (b) $\Rightarrow(\mathrm{e})$ in Theorem 13 of $[20]$.

As a third example: Some earlier theorems can be derived more efficiently using Theorem 1: Combined results of [19] (Theorem 24) and [8] (Theorem 6.1) show that $\mathrm{S}_{1}(\Omega, \Omega) \Rightarrow \Omega \rightarrow(\Omega)_{k}^{n}$ for all $n$ and $k$. This can be derived as follows: By Theorem 2 of [20], $\mathrm{S}_{1}(\Omega, \Omega)$ implies that ONE has no winning strategy in $\mathrm{G}_{1}(\Omega, \Omega)$. By Corollary 2 the partition relation $\Omega \rightarrow(\Omega)_{k}^{n}$ holds for all $n$ and $k$. Theorem 23 of [22] can be derived similarly since $\mathcal{D}_{\Omega}$ is persistent.

Similarly, if for a family $\mathcal{B}$ ONE has no winning strategy in the game $\mathrm{G}_{\mathrm{fin}}(\Omega, \mathcal{B})$, then by Theorem 3 the partition relation $\Omega \rightarrow\lceil\mathcal{B}\rceil_{k}^{2}$ holds for each finite $k$.

Thus Theorem 3 gives the implication $1 \Rightarrow 2$ in Theorem 6 of [24], as well as the implication (b) $\Rightarrow(f)$ of Theorem 11 of [20] and the implication $1 \Rightarrow 2$ in Theorem 10 of $[22]$.

From partition relations to selection principles. The reader could verify the following lemmas:

Lemma 12. Let $X$ be an $\epsilon$-space. Then $(\Omega, \Omega),(\Omega, \Lambda),(\Lambda, \Lambda),\left(\Omega, \Omega^{\mathrm{gp}}\right)$, $\left(\Omega^{\mathrm{gp}}, \Omega^{\mathrm{gp}}\right),\left(\Omega^{\mathrm{gp}}, \Omega\right),\left(\Omega, \Lambda^{\mathrm{gp}}\right),\left(\Lambda^{\mathrm{gp}}, \Lambda^{\mathrm{gp}}\right),\left(\Lambda^{\mathrm{gp}}, \Lambda\right)$ and $\left(\Lambda, \Lambda^{\mathrm{gp}}\right)$ are selectable pairs. 
LEMMA 13. If for a space $X$ each element of $\mathcal{D}_{\Omega}$ has a countable subset in $\mathcal{D}_{\Omega}$ then $\left(\mathcal{D}_{\Omega}, \mathcal{D}_{\Omega}\right)$ and $\left(\mathcal{D}_{\Omega}, \mathcal{D}\right)$ are selectable pairs.

The implication $2 \Rightarrow 1$ in Theorem 4 of [24] follows directly from Theorem 5 and the fact that $(\Omega, \Lambda)$ is a selectable pair, and similarly the implication $2 \Rightarrow 1$ of Theorem 6 of [24] follows from Theorem 4. Similarly, the implication $2 \Rightarrow 1$ in Theorem 23 of [22] is derived from Theorem 5 while $2 \Rightarrow 1$ in Theorem 10 of [22] is derived from Theorem 4 .

Note however that even when $X$ has countable tightness at $x$ the pair $\left(\Omega_{x}, \Omega_{x}\right)$ need not be a selectable pair. Different techniques were used in [20] to derive the selection principles there from the corresponding Ramseyan partition relations.

5. Characterizing the Hurewicz property. As mentioned in the introduction, several classical properties have been characterized (or defined) by selection principles of the form $\mathrm{S}_{\mathrm{fin}}(\mathcal{A}, \mathcal{B})$ or $\mathrm{S}_{1}(\mathcal{A}, \mathcal{B})$. No analogues of these were known for the Hurewicz property. A careful examination of the groupability property has led us to a characterization of the Hurewicz property in terms of a simple selection principle of the form $\mathrm{S}_{\mathrm{fin}}(\mathcal{A}, \mathcal{B})$.

TheOREM 14. For an $\epsilon$-space $X$ the following are equivalent:

(1) X has the Hurewicz property.

(2) $X$ has the Menger property $\mathrm{S}_{\text {fin }}(\Lambda, \Lambda)$ and each countable large cover of $X$ is groupable.

(3) $X$ has the property $\mathrm{S}_{\mathrm{fin}}(\Omega, \Lambda)$ and each countable large cover of $X$ is groupable.

(4) $X$ has property $\mathrm{S}_{\mathrm{fin}}\left(\Lambda, \Lambda^{\mathrm{gp}}\right)$.

(5) $X$ has property $\mathrm{S}_{\mathrm{fin}}\left(\Omega, \Lambda^{\mathrm{gp}}\right)$.

(6) On $X$ ONE has no winning strategy in the game $\mathrm{G}_{\mathrm{fin}}\left(\Omega, \Lambda^{\mathrm{gp}}\right)$.

(7) $X$ satisfies: For each $n, \Omega \rightarrow\left\lceil\Lambda^{\mathrm{gp}}\right\rceil_{n}^{2}$.

Proof. $(1) \Rightarrow(2)$. It is well known that the Hurewicz property implies $\mathrm{S}_{\mathrm{fin}}(\Lambda, \Lambda)$ - see for example [8]. And by Lemma 7 each countable large cover of $X$ is groupable.

$(2) \Rightarrow(3)$. Since each $\omega$-cover is a large cover, $\mathrm{S}_{\mathrm{fin}}(\Lambda, \Lambda)$ implies $\mathrm{S}_{\mathrm{fin}}(\Omega, \Lambda)$.

$(2) \Rightarrow(4)$. Let a sequence $\left(\mathcal{U}_{n}: n<\infty\right)$ of large covers of $X$ be given. We may assume that these are countable. Applying $\mathrm{S}_{\mathrm{fin}}(\Lambda, \Lambda)$ we find for each $n$ a finite set $\mathcal{V}_{n} \subset \mathcal{U}_{n}$ such that $\bigcup_{n<\infty} \mathcal{V}_{n}$ is a large cover of $X$. Since this large cover of $X$ is countable, it is groupable.

$(3) \Rightarrow(5)$. This is like $(2) \Rightarrow(4)$.

$(4) \Rightarrow(5)$. Since an $\omega$-cover is large, this implication is self-evident. 
$(5) \Rightarrow(1)$. Let a sequence $\left(\mathcal{U}_{n}: n<\infty\right)$ of open covers of $X$ be given. We may assume that these are countable, and that none contains a finite cover of $X$.

Replace each $\mathcal{U}_{n}$ with $\mathcal{V}_{n}$, the set of finite unions of elements of $\mathcal{U}_{n}$. Then each $\mathcal{V}_{n}$ is an $\omega$-cover of $X$. Enumerate each $\mathcal{V}_{n}$ bijectively as $\left(V_{k}^{n}: k<\infty\right)$. From these $\omega$-covers define new $\omega$-covers $\mathcal{W}_{n}$ as follows: $\mathcal{W}_{1}=\mathcal{V}_{1}$, and for $n>1, \mathcal{W}_{n}=\left\{V_{m_{1}}^{1} \cap V_{m_{2}}^{2} \cap \ldots \cap V_{m_{n}}^{n}: n<m_{1}<m_{2}<\ldots<m_{n}\right\} \backslash\{\emptyset\}$, and for each element of $\mathcal{W}_{n}$ choose a representation, fixed for the duration of the argument, of the form $V_{m_{1}}^{1} \cap V_{m_{2}}^{2} \cap \ldots \cap V_{m_{n}}^{n}$ with $n<m_{1}<m_{2}<\ldots<m_{n}$.

Apply $\mathrm{S}_{\text {fin }}\left(\Omega, \Lambda^{\text {gp }}\right)$ to the sequence $\left(\mathcal{W}_{n}: n<\infty\right)$ to find for each $n$ a finite set $\mathcal{X}_{n} \subset \mathcal{W}_{n}$ such that $\bigcup_{n<\infty} \mathcal{X}_{n}$ is a groupable large cover of $X$. Choose for each $n$ a finite set $\mathcal{Y}_{n}$ such that these are disjoint from each other, $\bigcup_{n<\infty} \mathcal{X}_{n}=\bigcup_{n<\infty} \mathcal{Y}_{n}$, and each element of $X$ belongs to all but finitely many of the sets $\cup \mathcal{Y}_{n}$.

Choose $n_{1}>1$ so large that $\mathcal{Y}_{n_{1}} \subset \bigcup_{j>1} \mathcal{X}_{j}$. Then let $\mathcal{Z}_{1}$ be the set of $V_{k}^{1}$ that appear as terms in the chosen representations of elements of $\mathcal{Y}_{n_{1}}$. Then choose $n_{2}>n_{1}$ so large that $\mathcal{Y}_{n_{2}} \subset \bigcup_{j>2} \mathcal{X}_{j}$ and let $\mathcal{Z}_{2}$ be the set of $V_{k}^{2}$ that appear as terms in the chosen representations of elements of $\mathcal{Y}_{n_{2}}$, and so on. In this way we obtain finite sets $\mathcal{Z}_{n} \subset \mathcal{V}_{n}$ such that each element of $X$ belongs to all but finitely many of the sets $\bigcup \mathcal{Z}_{n}$.

Finally, for each element $A$ of $\mathcal{Z}_{n}$ choose finitely many elements of $\mathcal{U}_{n}$ whose union produces $A$ and let $\mathcal{A}_{n}$ denote the finite set of elements of $\mathcal{U}_{n}$ chosen in this way. Then the sequence $\left(\mathcal{A}_{n}: n<\infty\right)$ witnesses the Hurewicz property of $X$ for the given sequence $\left(\mathcal{U}_{n}: n<\infty\right)$ of open covers.

$(1) \Rightarrow(6)$. Let $\tau$ be a strategy for ONE in the game $\mathrm{G}_{\mathrm{fin}}\left(\Omega, \Lambda^{\mathrm{gp}}\right)$. Since we are assuming that $X$ has the Hurewicz property, we know ONE has no winning strategy in the Hurewicz game. Use $\tau$ to define a strategy $\sigma$ for ONE of the Hurewicz game as follows:

$\sigma(\emptyset)=\tau(\emptyset)$. If TWO responds with $T_{1}$ in the Hurewicz game, then ONE first computes $\tau\left(T_{1}\right)$, and then plays $\sigma\left(T_{1}\right)=\tau\left(T_{1}\right) \backslash T_{1}$. If TWO responds in the Hurewicz game with $T_{2}$, then ONE first computes $\tau\left(T_{1}, T_{2}\right)$, and then responds with $\sigma\left(T_{1}, T_{2}\right)=\tau\left(T_{1}, T_{2}\right) \backslash\left(T_{1} \cup T_{2}\right)$, and so on. Observe that $\sigma$ is a legitimate strategy for ONE of the Hurewicz game - in fact, it directs ONE to play $\omega$-covers.

Since $\sigma$ is not a winning strategy for ONE of the Hurewicz game, consider a $\sigma$-play lost by ONE: $\sigma(\emptyset), T_{1}, \sigma\left(T_{1}\right), T_{2}, \sigma\left(T_{1}, T_{2}\right), T_{3}, \ldots$ By the definition of $\sigma$ the $T_{n}$ 's are disjoint from each other. Since TWO wins, each element of $X$ belongs to all but finitely many of the $\cup T_{n}$ 's. Thus, $\left(T_{n}: n<\infty\right)$ witnesses that $\bigcup_{n<\infty} T_{n}$ is a groupable cover of $X$.

Since also $\tau(\emptyset), T_{1}, \tau\left(T_{1}\right), T_{2}, \tau\left(T_{1}, T_{2}\right), T_{3}, \ldots$ is a legitimate play of $\mathrm{G}_{\text {fin }}\left(\Omega, \Lambda^{\mathrm{gp}}\right)$, we conclude that $\tau$ is not a winning strategy for ONE. 
$(6) \Rightarrow(7)$. Apply Theorem 3 and the fact that $\Omega$ is a persistent family.

$(7) \Rightarrow(5)$. By Lemma 12 the pair $\left(\Omega, \Lambda^{\mathrm{gp}}\right)$ is selectable. Now apply Theorem 4 .

The point of the following lemma is that one can under its hypotheses find for a given sequence of $\omega$-covers a selector which is itself a witness of the groupability of the $\omega$-cover constituted by the selector.

Lemma 15. Let $X$ satisfy both $\mathrm{S}_{\text {fin }}(\Omega, \Omega)$ and $\Omega=\Omega^{\mathrm{gp}}$. Then there is for each sequence $\left(\mathcal{U}_{n}: n<\infty\right)$ of $\omega$-covers of $X$ a sequence $\left(\mathcal{V}_{n}: n<\infty\right)$ such that:

(1) For each $n, \mathcal{V}_{n}$ is a finite subset of $\mathcal{U}_{n}$.

(2) For $m \neq n, \mathcal{V}_{m} \cap \mathcal{V}_{n}=\emptyset$.

(3) For each finite subset $F$ of $X$, for all but finitely many $n$ there is a $V \in \mathcal{V}_{n}$ such that $F \subset V$.

Proof. Let $\left(\mathcal{U}_{n}: n<\infty\right)$ be a sequence of $\omega$-covers of $X$. Since $X$ has the property that $\Omega=\Omega^{\text {gp }}$ we may by Lemma 9 assume that the $\mathcal{U}_{n}$ 's are disjoint from each other. By $\mathrm{S}_{\text {fin }}(\Omega, \Omega)$ we may also assume that each $\mathcal{U}_{n}$ is countable. Enumerate each $\mathcal{U}_{n}$ bijectively as $\left(U_{k}^{n}: k<\infty\right)$.

For each $n$ define $\mathcal{V}_{n}$ to be the collection of nonempty sets of the form

$$
U_{m_{1}}^{1} \cap \ldots \cap U_{m_{n}}^{n}
$$

Then each $\mathcal{V}_{n}$ is an $\omega$-cover of $X$. Apply $\mathrm{S}_{\text {fin }}(\Omega, \Omega)$ to this sequence to find for each $n$ a finite nonempty set $\mathcal{V}_{n}^{\prime} \subset \mathcal{V}_{n}$ such that $\mathcal{V}=\bigcup_{n<\infty} \mathcal{V}_{n}^{\prime}$ is an $\omega$-cover of $X$.

Applying $\Omega=\Omega^{\text {gp }}$ again, select a partition $\mathcal{V}=\bigcup_{k<\infty} \mathcal{W}_{k}$ of $\mathcal{V}$ such that each $\mathcal{W}_{k}$ is finite, and for each finite subset $F$ of $X$, for all but finitely many $k$ there is a $W \in \mathcal{W}_{k}$ such that $F \subset W$. Put $k_{1}=1$ and let $\mathcal{H}_{1}$ be the set of $U_{i}^{1}$ 's that occur as terms in the chosen representations of elements of $\mathcal{W}_{k_{1}}$. Next choose $k_{2}>k_{1}$ so large that for all $j \geq k_{2}$ we have $\mathcal{W}_{j}$ disjoint from $\mathcal{V}_{1}^{\prime}$. Let $\mathcal{H}_{2}$ consist of all sets of the form $U_{i}^{2}$ that occur as a term in the chosen representation of an element of $\mathcal{W}_{k_{2}}$. Then choose $k_{3}>k_{2}$ so large that for all $j \geq k_{3}$ we have $\mathcal{W}_{j} \cap \mathcal{V}_{2}^{\prime}=\emptyset$, and let $\mathcal{H}_{3}$ consist of the $U_{i}^{3}$ 's that occur as terms in the chosen representations of elements of $\mathcal{W}_{k_{3}}$, and so on.

In this way we obtain a sequence $\left(\mathcal{H}_{n}: n<\infty\right)$ of finite sets as required.

TheORem 16. For an $\epsilon$-space $X$ the following are equivalent:

(1) Each finite power of $X$ has the Hurewicz property.

(2) $X$ has property $\mathrm{S}_{\text {fin }}(\Omega, \Omega)$ and $\Omega=\Omega^{\text {gp }}$.

(3) $X$ has property $\mathrm{S}_{\text {fin }}\left(\Omega, \Omega^{\mathrm{gp}}\right)$.

(4) ONE has no winning strategy in the game $\mathrm{G}_{\mathrm{fin}}\left(\Omega, \Omega^{\mathrm{gp}}\right)$.

(5) For each $n, X$ satisfies $\Omega \rightarrow\left\lceil\Omega^{\mathrm{gp}}\right\rceil_{n}^{2}$. 
Proof. (1) $\Rightarrow(2)$. If each finite power of $X$ has the Hurewicz property, then each finite power of $X$ has property $\mathrm{S}_{\text {fin }}(\mathcal{O}, \mathcal{O})$. Then Theorem 3.9 of [8] implies that $X$ has property $\mathrm{S}_{\text {fin }}(\Omega, \Omega)$. Lemma 5 of [9] implies that $X$ satisfies $\Omega=\Omega^{\text {gp }}$.

$(2) \Leftrightarrow(3)$. This is clear.

$(2) \Rightarrow(4)$. Let $\sigma$ be a strategy for ONE in the game $\mathrm{G}_{\text {fin }}\left(\Omega, \Omega^{\mathrm{gp}}\right)$. Then it is also a strategy in $\mathrm{G}_{\text {fin }}(\Omega, \Omega)$. By $(2), X$ has property $\mathrm{S}_{\text {fin }}(\Omega, \Omega)$ and so by Theorem 5 of $[20]$ this is not a winning strategy for $\operatorname{ONE}$ in $\mathrm{G}_{\mathrm{fin}}(\Omega, \Omega)$. Consider a $\sigma$-play which is lost by ONE in $\mathrm{G}_{\text {fin }}(\Omega, \Omega)$. Then TWO's moves constitute an $\omega$-cover of $X$. Again by (2), it is groupable. Thus, this play is actually lost by $\mathrm{ONE}$ in the game $\mathrm{G}_{\mathrm{fin}}\left(\Omega, \Omega^{\mathrm{gp}}\right)$.

$(4) \Rightarrow(5)$. Apply Theorem 3 .

$(5) \Rightarrow(2)$. Since $X$ satisfies $\Omega \rightarrow\left\lceil\Omega^{\mathrm{gp}}\right\rceil_{k}^{2}$ for each $k$, it also satisfies $\Omega \rightarrow$ $\lceil\Omega\rceil_{k}^{2}$ for each $k$. By Theorem 6.2 of $[8], X$ then has property $\mathrm{S}_{\text {fin }}(\Omega, \Omega)$. Moreover, the partition relation implies that each $\omega$-cover contains a groupable $\Omega$-cover.

$(2) \Rightarrow(1)$. Fix $n$ and let $\left(\mathcal{U}_{k}: k<\infty\right)$ be a sequence of large covers of $X^{n}$. For each $k$ let $\mathcal{V}_{k}$ be the collection of open subsets $V$ of $X$ such that $V^{n}$ is a subset of a union of finitely many elements of $\mathcal{U}_{k}$. Then each $\mathcal{V}_{k}$ is an $\omega$-cover of $X$ : For let a finite subset $F$ of $X$ be given. Let $\mathcal{U}_{F, k}$ be a finite subset of $\mathcal{U}_{k}$ which covers $F^{n}$. Since $F^{n}$ is compact Wallace's theorem implies that there is an open set $V \subset X$ such that $F^{n} \subset V^{n} \subset \cup \mathcal{U}_{F, k}$. Thus, $F \subset V$ and $V \in \mathcal{V}_{k}$. Apply (2) and Lemma 15 and choose for each $k$ a finite set $\mathcal{V}_{k}^{\prime} \subset \mathcal{V}_{k}$ such that the sequence $\left(\mathcal{V}_{k}^{\prime}: k<\infty\right)$ has the properties of Lemma 15 . For each $k$ choose for each element $V$ of $\mathcal{V}_{k}^{\prime}$ finitely many elements of $\mathcal{U}_{k}$ which cover $V^{n}$, and let $\mathcal{W}_{k}$ be the finite set of elements of $\mathcal{U}_{k}$ chosen like this. Then the sequence $\left(\mathcal{W}_{k}: k<\infty\right)$ witnesses the Hurewicz property in $X^{n}$ for $\left(\mathcal{U}_{k}: k<\infty\right)$.

Next we give a new characterization of the cardinal number $\mathfrak{b}$ - the least cardinality of an unbounded family in ${ }^{\mathbb{N}} \mathbb{N}$. A combinatorial $\omega$-cover for a set $S$ is a collection of subsets of $S$ such that each finite subset of $S$ is a subset of some member of the collection, but $S$ itself is not a member of the collection. Thus, for combinatorial open covers we do not require an ambient topology. For $\omega$-covers (no additional adjective) we require that the members of the cover be open sets.

For a cardinal number $\kappa$ we let $\Omega_{\kappa}$ denote the collection of countable combinatorial $\omega$-covers of $\kappa$. Also, we let $\Omega_{\kappa}^{\mathrm{gp}}$ denote the groupable combinatorial $\omega$-covers of $\kappa$.

THEOREM 17. For an infinite cardinal number $\kappa$ the following are equivalent: 
(1) $\kappa<\mathfrak{b}$.

(2) $\mathrm{S}_{\mathrm{fin}}\left(\Omega_{\kappa}, \Omega_{\kappa}\right)$ and $\Omega_{\kappa}=\Omega_{\kappa}^{\mathrm{gp}}$ hold.

(3) $\mathrm{S}_{\text {fin }}\left(\Omega_{\kappa}, \Omega_{\kappa}^{\mathrm{gp}}\right)$ holds.

(4) ONE has no winning strategy in the game $\mathrm{G}_{\mathrm{fin}}\left(\Omega_{\kappa}, \Omega_{\kappa}^{\mathrm{gp}}\right)$.

(5) For each $n, \Omega_{\kappa} \rightarrow\left\lceil\Omega_{\kappa}^{\mathrm{gp}}\right\rceil_{n}^{2}$ holds.

Proof. $(1) \Rightarrow(2)$. First we show that (1) implies that $\Omega_{\kappa}=\Omega_{\kappa}^{\mathrm{gp}}$. Thus, let $\mathcal{U}$ be an element of $\Omega_{\kappa}$, and enumerate it bijectively as $\left(U_{n}: n<\infty\right)$. For each finite subset $F$ of $\kappa$, define a function $\Phi_{F}$ from $\mathbb{N}$ to $\mathbb{N}$ such that for each $n, \Phi_{F}(n)=\min \left\{k>n: F \subset U_{k}\right\}$.

Then $\left\{\Phi_{F}: F \subset \kappa\right.$ finite $\}$ is a family of fewer than $\mathfrak{b}$ functions. Choose an increasing function $g: \mathbb{N} \rightarrow \mathbb{N}$ such that $1<g(1)$, and for each finite subset $F$ of $\kappa$, for all but finitely many $n$ we have $\Phi_{F}(n)<g(n)$. Then put $n_{1}=g(1)$, and for each $k$ put $n_{k+1}=g\left(n_{k}\right)$. Observe that now for each finite $F \subset \kappa$, for all but finitely many $k$, we have $n_{k}<\Phi_{F}\left(n_{k}\right)<n_{k+1}$. Thus, if we set $\mathcal{V}_{1}=\left\{U_{j}: j<n_{1}\right\}$ and $\mathcal{V}_{k+1}=\left\{U_{j}: n_{k} \leq j<n_{k+1}\right\}$ for each $k$, then we have a partition of $\mathcal{U}$ into pairwise disjoint finite sets such that for each finite $F \subset \kappa$, for all but finitely many $k$, there is a $V \in \mathcal{V}_{k}$ such that $F \subset V$.

Next we show that (1) implies $\mathrm{S}_{\text {fin }}\left(\Omega_{\kappa}, \Omega_{\kappa}\right)$. Let $\left(\mathcal{U}_{n}: n<\infty\right)$ be a sequence of elements of $\Omega_{\kappa}$. By what we have just proved, choose for each $n$ a sequence $\left(\mathcal{U}_{k}^{n}: k<\infty\right)$ of pairwise disjoint finite subsets of $\mathcal{U}_{n}$ such that for each finite $F \subset \kappa$, for all but fintely many $k$ there is a $V \in \mathcal{U}_{k}^{n}$ such that $F \subset V$. Next, define for each finite set $F \subset \kappa$ a function $\Psi: \mathbb{N} \rightarrow \mathbb{N}$ so that for each $n, \Psi_{F}(n)$ is the least $k$ such that for each $j \geq k$ there is a $V \in \mathcal{U}_{j}^{n}$ with $F \subset V$.

Since $\left\{\Psi_{F}: F \subset \kappa\right.$ finite $\}$ is a family of fewer than $\mathfrak{b}$ such functions, choose an increasing function $g$ from $\mathbb{N}$ to $\mathbb{N}$ such that for each finite $F \subset \kappa$, for all but finitely many $n, \Psi_{F}(n)<g(n)$. Then define $\mathcal{V}_{k}=\mathcal{U}_{g(k)}^{k}$ for each $k$. Then $\bigcup_{k<\infty} \mathcal{V}_{k}$ is a member of $\Omega_{\kappa}$.

$(2) \Rightarrow(3)$. This is left as an exercise.

$(3) \Rightarrow(1)$. Let $X$ be a set of real numbers of cardinality $\kappa$ and let $\left(\mathcal{U}_{n}\right.$ : $n<\infty)$ be a sequence of $\omega$-covers of $X$. Since each finite power of $X$ is Lindelöf, we may assume that each $\mathcal{U}_{n}$ is countable, and thus a member of $\Omega_{\kappa}$. Now apply (3) to choose from each $\mathcal{U}_{n}$ a finite set $\mathcal{V}_{n}$ such that $\bigcup_{n<\infty} \mathcal{V}_{n}$ is in $\Omega_{\kappa}^{\mathrm{gp}}$. Then in fact $\bigcup_{n<\infty} \mathcal{V}_{n}$ is in $\Omega^{\mathrm{gp}}$. This shows that each set of real numbers of cardinality $\kappa$ has the property $\mathrm{S}_{\text {fin }}\left(\Omega, \Omega^{\mathrm{gp}}\right)$, and thus the Hurewicz property. It follows from a result of Hurewicz that $\kappa<\mathfrak{b}$.

$(4) \Rightarrow(5)$. $\Omega_{\kappa}$ is persistent, so that this implication follows from Theorem 3 .

$(5) \Rightarrow(3)$. The pair $\left(\Omega_{\kappa}, \Omega_{\kappa}\right)$ is selectable, and so this implication follows from Theorem 4. 
It remains to show that (1) implies (4). In this proof we also use the fact that (1) is equivalent to (2). Let $\sigma$ be a strategy for ONE of the game $\mathrm{G}_{\mathrm{fin}}\left(\Omega_{\kappa}, \Omega_{\kappa}^{\mathrm{gp}}\right)$. We shall assume that TWO's moves are "initial segments" of ONE's, in the following sense:

Enumerate ONE's first move, $\sigma(\emptyset)$, bijectively as $\left(U_{n}: n<\infty\right)$. For any $n_{1}$, enumerate $\sigma\left(\left\{U_{j}: j<n_{1}\right\}\right)$ bijectively as $\left(U_{n_{1}, n}: n<\infty\right)$ (thus, we pretend TWO responded with $\left.\left\{U_{j}: j<n_{1}\right\}\right)$. For any $n_{2}$, enumerate $\sigma\left(\left\{U_{i}: i<n_{1}\right\},\left\{U_{n_{1}, j}: j<n_{2}\right\}\right)$ bijectively as $\left(U_{n_{1}, n_{2}, j}: j<\infty\right)$, and so on.

For each finite set $F \subset \kappa$, and for each finite sequence $\tau$ of positive integers, define $\Psi_{F}(\tau)$ to be the least $k$ such that $\max (\tau)<k$ and $F \subset U_{\tau \frown k}$. Since by (1) we have $\kappa<\mathfrak{b}$, we find a function $g$ from the set of finite sequences of elements of $\mathbb{N}$ to $\mathbb{N}$ such that for each finite subset $F$ of $\kappa$ and for all but finitely many $\tau$ we have $g(\tau)>\Psi_{F}(\tau)$.

Put $n_{1}=g(1)$, and for each $k, n_{k+1}=g\left(n_{1}, \ldots, n_{k}\right)$.

For each finite $F \subset \kappa$, for all but finitely many $k$ we have $\Psi_{F}\left(n_{1}, \ldots, n_{k-1}\right)$ $<n_{k}$. To see this, fix $F$. Since the set of $\tau_{k}=\left(n_{1}, \ldots, n_{k}\right), k<\infty$ is infinite, for all but finitely many $k$ we have $\Psi_{F}\left(\tau_{k}\right)<g\left(\tau_{k}\right)=n_{k+1}$.

This means that the $\sigma$-play during which TWO chose consecutively the sets $T_{1}=\left\{U_{j}: j<n_{1}\right\}, \ldots, T_{k}=\left\{U_{n_{1}, \ldots, n_{k-1}, j}: j<n_{k}\right\}, \ldots$ is lost by ONE since $\bigcup\left\{T_{j}: j<\infty\right\}$ is a member of $\Omega_{\kappa}$, and by (2) it is groupable.

6. Characterizing the Gerlits-Nagy property. In [5] Gerlits and Nagy introduced a property denoted $(*)$. Property $(*)$ was characterized in [13] as follows: $X$ has property $(*)$ if, and only if, it has Hurewicz's property as well as property $\mathrm{S}_{1}(\mathcal{O}, \mathcal{O})$. We shall take this characterization as our official definition of the Gerlits-Nagy property.

Theorem 18. For an $\epsilon$-space $X$ the following are equivalent:

(1) $X$ has the Gerlits-Nagy property.

(2) $X$ has property $\mathrm{S}_{1}\left(\Lambda, \Lambda^{\mathrm{gp}}\right)$.

(3) $X$ has property $\mathrm{S}_{1}\left(\Omega, \Lambda^{\mathrm{gp}}\right)$.

(4) On $X$ ONE has no winning strategy in the game $\mathrm{G}_{1}\left(\Omega, \Lambda^{\mathrm{gp}}\right)$.

(5) For each $n, X$ satisfies $\Omega \rightarrow\left(\Lambda^{\text {gp }}\right)_{n}^{2}$.

Proof. $(1) \Rightarrow(2)$. Since $X$ has property $\mathrm{S}_{1}(\mathcal{O}, \mathcal{O})$ it also has property $\mathrm{S}_{1}(\Lambda, \Lambda)$ (by the proof of Theorem 17 of [19]). Since $X$ has the Hurewicz property, every countable large cover of $X$ is groupable (Lemma 7 ). It follows that $X$ has property $\mathrm{S}_{1}\left(\Lambda, \Lambda^{\mathrm{gp}}\right)$.

$(2) \Rightarrow(3)$. Every $\omega$-cover is large.

$(3) \Rightarrow(1)$. Since $X$ has property $\mathrm{S}_{1}\left(\Omega, \Lambda^{\mathrm{gp}}\right)$, it has property $\mathrm{S}_{1}(\Omega, \Lambda)$ and thus property $\mathrm{S}_{1}(\mathcal{O}, \mathcal{O})$ (Theorem 17 of [19]). Since $\mathrm{S}_{1}\left(\Omega, \Lambda^{\mathrm{gp}}\right)$ implies $\mathrm{S}_{\text {fin }}\left(\Omega, \Lambda^{\mathrm{gp}}\right)$, Theorem 14 implies that $X$ has the Hurewicz property. 
$(1) \Rightarrow(4)$. By Theorem 3 of [24], ONE has no winning strategy in the game $\mathrm{G}_{1}(\Omega, \Lambda)$. Let $F$ be a strategy for $\mathrm{ONE}$ in $\mathrm{G}_{1}\left(\Omega, \Lambda^{\mathrm{gp}}\right)$. Then $F$ is also a strategy for ONE in the game $\mathrm{G}_{1}(\Omega, \Lambda)$. Since ONE has no winning strategy in the latter game, consider an $F$-play

$$
F(\emptyset), T_{1}, F\left(T_{1}\right), T_{2}, F\left(T_{1}, T_{2}\right), T_{3}, \ldots
$$

which is lost by ONE in $\mathrm{G}_{1}(\Omega, \Lambda)$. Then $\left\{T_{n}: n<\infty\right\}$ is a large cover of $X$. Since $X$ has the Hurewicz property, this large cover of $X$ is groupable (Lemma 7 ). But then the given $F$-play is also lost by $\mathrm{ONE}$ in $\mathrm{G}_{1}\left(\Omega, \Lambda^{\mathrm{gp}}\right)$.

$(4) \Rightarrow(5)$. Since $\Omega$ is a persistent family, apply Theorem 1 .

$(5) \Rightarrow(3)$. Since $\left(\Omega, \Lambda^{\mathrm{gp}}\right)$ is a selectable pair (Lemma 12$)$, apply Theorem 5 .

The Gerlits-Nagy property in all finite powers is the main covering property featured in [9]. We now characterize this property by a Ramseyan partition relation:

TheOREM 19. For an $\epsilon$-space $X$ the following are equivalent:

(1) Each finite power of $X$ has the Gerlits-Nagy property.

(2) $X$ has property $\mathrm{S}_{1}(\Omega, \Omega)$ and $\Omega=\Omega^{\mathrm{gp}}$.

(3) $X$ has property $\mathrm{S}_{1}\left(\Omega, \Omega^{\mathrm{gp}}\right)$.

(4) On $X$ ONE has no winning strategy in the game $\mathrm{G}_{1}\left(\Omega, \Omega^{\mathrm{gp}}\right)$.

(5) For each $n$ and $k, X$ satisfies $\Omega \rightarrow\left(\Omega^{\mathrm{gp}}\right)_{k}^{n}$.

Proof. The argument follows similar lines to that for Theorem 18, but uses Theorem 16 instead of 14, Lemma 8 instead of 7, and Theorem 2 of [20] instead of Theorem 3 of [24].

Next we give new characterizations of the cardinal number $\operatorname{add}(\mathcal{M})$. This cardinal is the least cardinality of a family of first category sets whose union is not first category. The $\operatorname{symbol} \operatorname{cov}(\mathcal{M})$ denotes the least cardinality of a family of first category sets whose union is the real line. Miller has shown that $\operatorname{add}(\mathcal{M})=\min \{\operatorname{cov}(\mathcal{M}), \mathfrak{b}\}$. It was shown in [21] that the following are equivalent for an infinite cardinal $\kappa$ :

1. $\kappa<\operatorname{cov}(\mathcal{M})$.

2. For each $n$ and $k, \Omega_{\kappa} \rightarrow\left(\Omega_{\kappa}\right)_{k}^{n}$.

We shall use this information below. Also, note that $\Omega_{\kappa}$ is a persistent family, and the pair $\left(\Omega_{\kappa}, \Omega_{\kappa}^{\mathrm{gp}}\right)$ is selectable.

THEOREM 20. For an infinite cardinal number $\kappa$ the following are equivalent:

(1) $\kappa<\operatorname{add}(\mathcal{M})$.

(2) ONE has no winning strategy in the game $\mathrm{G}_{1}\left(\Omega_{\kappa}, \Omega_{\kappa}^{\mathrm{gp}}\right)$. 
(3) For each $n$ and $k, \Omega_{\kappa} \rightarrow\left(\Omega_{\kappa}^{\mathrm{gp}}\right)_{k}^{n}$.

(4) $\mathrm{S}_{1}\left(\Omega_{\kappa}, \Omega_{\kappa}^{\mathrm{gp}}\right)$ holds.

Proof. $(1) \Rightarrow(2)$. Let $F$ be a strategy for ONE in the game. Define a family $\left(U_{n_{1}, n_{2}, \ldots, n_{k}}: n_{1}, \ldots, n_{k}<\infty\right)$ of subsets of $\kappa$ as follows: ONE's first move is $F(\emptyset)$, a countable combinatorial $\omega$-cover of $\kappa$; enumerate it bijectively by $\left(U_{n}: n<\infty\right)$. If TWO chose $U_{n_{1}} \in F(\emptyset)$, then ONE's response is another countable combinatorial $\omega$-cover of $\kappa$-enumerate it bijectively as $\left(U_{n_{1}, n}: n<\infty\right)$, and so on.

The family $\left(U_{n_{1}, \ldots, n_{k}}: n_{1}, \ldots, n_{k}<\infty\right)$ has the property that for each $\left(n_{1}, \ldots, n_{k}\right)$ the set $\left\{U_{n_{1}, \ldots, n_{k}, n}: n<\infty\right\}$ is a countable, combinatorial $\omega$-cover of $\kappa$. For each nonempty finite subset $F$ of $\kappa$, define

$$
S_{F}=\left\{f \in{ }^{\mathbb{N}} \mathbb{N} \text { : for each } n, F \not \subset U_{f\left\lceil_{n}\right.}\right\} .
$$

Then each $S_{F}$ is a nowhere dense subset of ${ }^{\mathbb{N}} \mathbb{N}$ (where the topology is the product topology and $\mathbb{N}$ has the discrete topology). But ${ }^{\mathbb{N}} \mathbb{N}$ is homeomorphic to the space of irrational numbers, and so as $\kappa<\operatorname{add}(\mathcal{M})$ we find that

$$
\bigcup\left\{S_{F}: F \subset \kappa \text { finite }\right\}
$$

is first category. Choose an $f$ outside this union. Then the sequence

$$
F(\emptyset), U_{f(1)}, F\left(U_{f(1)}\right), U_{f(1), f(2)}, F\left(U_{f(1)}, U_{f(1), f(2)}\right), U_{f(1), f(2), f(3)}, \ldots
$$

shows that by the choice of $f,\left\{U_{f\left\lceil_{n}\right.}: n<\infty\right\}$ is a combinatorial $\omega$-cover of $\kappa$. We shall be done if we show that each countable combinatorial $\omega$-cover of $\kappa$ is groupable.

Thus, let $\mathcal{U}=\left\{U_{n}: n<\infty\right\}$ be a bijectively enumerated combinatorial $\omega$-cover of $\kappa$. For each nonempty finite subset $F$ of $\kappa$ define $f_{F}$ so that for each $n, f_{F}(n)$ is the least $m>f_{F}(n-1)$ for which there is a $j$ with $f_{F}(n)<j<m$ and $F \subset U_{j}$. Then $\left\{f_{F}: F \subset \kappa\right.$ finite $\}$ is a family of $\leq \kappa$ elements of ${ }^{\mathbb{N}} \mathbb{N}$. By Miller's theorem there is an increasing mapping $g: \mathbb{N} \rightarrow \mathbb{N}$ such that for each $F$, for all but finitely many $n$, we have $f_{F}(n)<g(n)$. We may assume $g(1)>1$.

Define $h$ by $h(n)=g^{\exp _{n+1}(2)}(1)$ for all $n$ (Here, $\exp _{0}(2)=2$, and $\left.\exp _{n+1}(2)=2^{\exp _{n}(2)}\right)$. Then for each nonempty finite $F \subset \kappa$, for all but finitely many $k$ there is a $j$ with $h(k)<f_{F}(j)<h(k+1)$. Thus, if for each $k$ we put $\mathcal{U}_{k}=\left\{U_{j}: h(k) \leq j<h(k+1)\right\}$ then the sequence $\left(\mathcal{U}_{k}: k<\infty\right)$ witnesses the groupability of the combinatorial $\omega$-cover $\mathcal{U}$ of $\kappa$.

$(2) \Rightarrow(3)$. Since $\Omega_{\kappa}$ is persistent, apply Theorem 1 .

$(3) \Rightarrow(4)$. Since $\left(\Omega_{\kappa}, \Omega_{\kappa}^{\mathrm{gp}}\right)$ is a selectable pair, apply Theorem 5 .

$(4) \Rightarrow(1)$. Consider a set $X$ of real numbers of cardinality $\kappa$. Since countable $\omega$-covers of $X$ are also combinatorial $\omega$-covers of $X$, (4) implies that $X$ has property $\mathrm{S}_{1}\left(\Omega, \Omega^{\mathrm{gp}}\right)$. Thus, each set of real numbers of cardinality $\kappa$ has the Gerlits-Nagy property. But in [13] it was shown that the least 
cardinality of a set of real numbers not having the Gerlits-Nagy property is $\operatorname{add}(\mathcal{M})$.

7. Countable fan tightness and the Reznichenko property in $\mathrm{C}_{\mathrm{p}}(X) . \mathrm{C}_{\mathrm{p}}(X)$ denotes the space of real-valued continuous functions on $X$, endowed with the topology of pointwise convergence. Since this space is homogeneous we may, when studying local properties, confine attention to any particular element of $\mathrm{C}_{\mathrm{p}}(X)$. For computational convenience we single out 0 , the constantly zero function. In [9] we characterized those $C_{p}(X)$ which have both the Reznichenko property and countable strong fan tightness in terms of classical covering properties of $X$. We now do the same for those $\mathrm{C}_{\mathrm{p}}(X)$ which have both the Reznichenko property and countable fan tightness.

TheOREM 21. For a Tikhonov space $X$ the following are equivalent:

(1) Each finite power of $X$ has the Hurewicz property.

(2) $\mathrm{C}_{\mathrm{p}}(X)$ has countable fan tightness as well as Reznichenko's property.

(3) On $\mathrm{C}_{\mathrm{p}}(X)$ ONE has no winning strategy in the game $\mathrm{G}_{\mathrm{fin}}\left(\Omega_{0}, \Omega_{0}^{\mathrm{gp}}\right)$.

(4) For each $k, \mathrm{C}_{\mathrm{p}}(X)$ satisfies $\Omega_{0} \rightarrow\left\lceil\Omega_{0}^{\mathrm{gp}}\right\rceil_{k}^{2}$.

(5) $\mathrm{C}_{\mathrm{p}}(X)$ has property $\mathrm{S}_{\mathrm{fin}}\left(\Omega_{0}, \Omega_{0}^{\mathrm{gp}}\right)$.

Proof. $(1) \Rightarrow(2)$. Since each finite power of $X$ has the Hurewicz property, each finite power of $X$ has the Menger property. By Theorem 3.9 of [8], $X$ has property $\mathrm{S}_{\mathrm{fin}}(\Omega, \Omega)$. By $[1], \mathrm{C}_{\mathrm{p}}(X)$ has countable fan tightness.

We must show that $\mathrm{C}_{\mathrm{p}}(X)$ has the Reznichenko property. Thus, let $A$ be an element of $\Omega_{0}$. We shall use the fact that ONE has no winning strategy in the game $\mathrm{G}_{\mathrm{fin}}\left(\Omega, \Omega^{\mathrm{gp}}\right.$ ) on $X$ (Theorem 16). Define the following strategy, $\sigma$, for $\mathrm{ONE}$ in $\mathrm{G}_{\mathrm{fin}}\left(\Omega, \Omega^{\mathrm{gp}}\right)$ : For each finite subset $F$ of $X$ the neighborhood $W(0, F, 1)$ of 0 has a nonempty intersection with $A$. Choose $f_{F} \in A$. Since $f_{F}$ is continuous, choose for each $x \in F$ an open set $V_{x}$ such that $f_{F}\left[V_{x}\right] \subset(-1,1)$, and then set $V_{F}=\bigcup_{x \in F} V_{x}$. ONE's first move is $\sigma(\emptyset)=\left\{V_{F}: F \subset X\right.$ finite $\}$, an $\omega$-cover of $X$. Supposing that the finite set $T_{1}=\left\{V_{F_{1}}, \ldots, V_{F_{k}}\right\}$ is TWO's response, ONE first associates with each element of TWO's response a corresponding function $f_{F_{j}} \in A$ for which $f_{F_{j}}\left[V_{F_{j}}\right] \subset(-1,1)$; let this finite set of functions be $B_{1}$. Then ONE considers $A \backslash B_{1}$, still an element of $\Omega_{0}$, and for each finite set $F \subset X$ chooses an $f_{F} \in A$ in the neighborhood $W(0, F, 1 / 2)$ of 0 , then for each $x$ in $F$ chooses a neighborhood $V_{x}$ with $f_{F}\left[V_{x}\right] \subset(-1 / 2,1 / 2)$, and finally defines $V_{F}=\bigcup_{x \in F} V_{x}$. Then ONE plays $\sigma\left(T_{1}\right)=\left\{V_{F}: F \subset X\right.$ finite $\} \backslash T_{1}$, an $\omega$-cover of $X$. Should TWO now choose a finite set $T_{2} \subset \sigma\left(T_{1}\right)$, then ONE selects for each $V_{F} \in T_{2}$ an $f_{F} \in A \backslash B_{1}$ for which $f_{F}\left[V_{F}\right] \subset(-1 / 2,1 / 2)$, and let $B_{2}$ be the finite set of functions thus selected. Then ONE considers $A \backslash\left(B_{1} \cup B_{2}\right)$, still an element of $\Omega_{0}$, and for each finite subset $F$ of $X$ chooses an $f_{F} \in A \backslash\left(B_{1} \cup B_{2}\right)$ 
which is in the neighborhood $W(0, F, 1 / 3)$ of 0 . For each $x \in F$ choose a neighborhood $V_{x}$ such that $f_{F}\left[V_{x}\right] \subset(-1 / 3,1 / 3)$, and put $V_{F}=\bigcup_{x \in F} V_{x}$. Then ONE plays the $\omega$-cover $\sigma\left(T_{1}, T_{2}\right)=\left\{V_{F}: F \subset X\right.$ finite $\} \backslash\left(T_{1} \cup T_{2}\right)$, and so on.

Since $\sigma$ is not a winning strategy for ONE, consider a $\sigma$-play lost by ONE, say

$$
\sigma(\emptyset), T_{1}, \sigma\left(T_{1}\right), T_{2}, \sigma\left(T_{1}, T_{2}\right), T_{3}, \ldots
$$

and let $B_{1}, B_{2}, \ldots$ be the corresponding disjoint finite subsets of $A$ constructed during the play. By the definition of $\sigma$ :

1. the sequence $\left(T_{1}, T_{2}, \ldots\right)$ is pairwise disjoint;

2 . the sequence $\left(B_{1}, B_{2}, \ldots\right)$ is pairwise disjoint;

3. for each $V_{F} \in T_{n}$ there is an $f \in B_{n}$ with $f\left[V_{F}\right] \subset(-1 / n, 1 / n)$.

Moreover, since ONE lost the play, $\bigcup_{n<\infty} T_{n}$ is a groupable $\omega$-cover of $X$. This implies that we may choose an infinite sequence $n_{1}<n_{2}<\ldots$ such that if we put $\mathcal{U}_{k}=\bigcup_{n_{k} \leq j<n_{k+1}} T_{j}$ for each $k$, then the $\mathcal{U}_{k}$ 's are disjoint from each other (because the $T_{n}$ 's are) and for each finite $F \subset X$, for all but finitely many $n$ there is a $V \in \mathcal{U}_{n}$ with $F \subset V$. Correspondingly define $C_{k}=\bigcup_{n_{k} \leq j<n_{k+1}} B_{j}$. Then the $C_{k}$ 's are disjoint finite sets. Let $W(0, F, 1 / n)$ be a neighborhood of 0 . For each $k$ for which this is possible, choose a $V_{k} \in \mathcal{U}_{k}$ with $F \subset V_{k}$, and then choose a corresponding $f_{k} \in C_{k}$ with $f_{k}\left[V_{k}\right] \subset(-1 / k, 1 / k)$. Since this can be done for all but finitely many $k$, we find that for all but finitely many $k$ we have $f_{k} \in W(0, F, 1 / n)$-i.e., for all but finitely many $k$ we have $C_{k} \cap W(0, F, 1 / n) \neq \emptyset$. Since $A$ is countable, any points in $A \backslash \bigcup_{k<\infty} C_{k}$ may be distributed among the $C_{k}$ so that after distribution each is still finite. Thus, we may assume that $A=\bigcup_{k<\infty} C_{k}$, completing the proof that $A$ is groupable. (Remark: we made the detour through a strategy of ONE in the game $\mathrm{G}_{\text {fin }}\left(\Omega, \Omega^{\mathrm{gp}}\right)$ on $X$ to ensure that the $B_{n}$ 's we came up with are disjoint from each other.)

$(2) \Rightarrow(3)$. Let $F$ be a strategy for ONE in the game $\mathrm{G}_{\text {fin }}\left(\Omega_{0}, \Omega_{0}^{\mathrm{gp}}\right)$ Then $F$ is also a strategy for ONE in the game $\mathrm{G}_{\mathrm{fin}}\left(\Omega_{0}, \Omega_{0}\right)$. Since $\mathrm{C}_{\mathrm{p}}(X)$ has countable fan tightness, Theorem 11 of [20] implies that ONE has no winnig strategy in the game $\mathrm{G}_{\text {fin }}\left(\Omega_{0}, \Omega_{0}\right)$. Consider a play lost by ONE, say $F(\emptyset), T_{1}, F\left(T_{1}\right), T_{2}, F\left(T_{1}, T_{2}\right), \ldots$ Then $\bigcup_{n<\infty} T_{n}$ is a countable member of $\Omega_{0}$. Since $\mathrm{C}_{\mathrm{p}}(X)$ also has the Reznichenko property, $\bigcup_{n<\infty} T_{n}$ is also groupable, and so this play is also lost by ONE in $\mathrm{G}_{\text {fin }}\left(\Omega_{0}, \Omega_{0}^{\mathrm{gP}}\right)$.

$(3) \Rightarrow(4)$. Since $\Omega_{0}$ is persistent, apply Theorem 3 .

$(4) \Rightarrow(5)$. Since $\Omega_{0} \rightarrow\left\lceil\Omega_{0}^{\mathrm{gp}}\right\rceil_{k}^{2}$ implies that each $A \in \Omega_{0}$ contains a countable $B \subset A$ with $B \in \Omega_{0}^{\mathrm{gp}}$, it follows that each countable element of $\Omega_{0}$ is groupable. Moreover, the partition relation also implies $\Omega_{0} \rightarrow\left\lceil\Omega_{0}\right\rceil_{k}^{2}$. By Theorem 11 of $[20]$ the latter implies that $C_{p}(X)$ has countable fan tightness, 
i.e., $\mathrm{S}_{\text {fin }}\left(\Omega_{0}, \Omega_{0}\right)$ holds. But since each countable element of $\Omega_{0}$ is groupable, this implies that $\mathrm{S}_{\text {fin }}\left(\Omega_{0}, \Omega_{0}^{\mathrm{gp}}\right)$ holds.

$(5) \Rightarrow(2)$. This is left as an exercise.

$(2) \Rightarrow(1)$. We show that $X$ satisfies $\mathrm{S}_{\text {fin }}\left(\Omega, \Omega^{\text {gp }}\right)$. Since $\mathrm{C}_{\mathrm{p}}(X)$ has countable fan tightness we know by a theorem of Arkhangel'skiu in [1] that finite powers of $X$ have the Menger property, and thus by Theorem 3.9 of [8] that $X$ has property $\mathrm{S}_{\text {fin }}(\Omega, \Omega)$. By Theorem 5 of [20], ONE has no winning strategy in the game $\mathrm{G}_{\text {fin }}(\Omega, \Omega)$ on $X$. We shall be done if we can show that each countable $\omega$-cover of $X$ is groupable. Let $\mathcal{U}$ be a countable $\omega$-cover of $X$.

For each finite set $F \subset X$ pick a $U \in \mathcal{U}$ with $F \subset U$, and let $f_{F, U}$ be a continuous function from $X$ to $[0,1]$ such that $f_{U, F}[F]=\{0\}$, and $f_{U, F}[X \backslash U]=\{1\}$. Then put $A=\left\{f_{U, F}: F \subset X\right.$ finite and $\left.U \in \mathcal{U}\right\}$. Then $A$ is in $\Omega_{0}$. We may assume that $A$ is countable (else, replace it by a countable subset still in $\Omega_{0}$ ). Correspondingly, we may thin out $\mathcal{U}$ so that it contains only those sets for which corresponding functions are in the thinned out $A$. Since $A$ is in $\Omega_{0}$ one can check that the thinned out $\mathcal{U}$ is still an $\omega$-cover of $X$. Thus, each element of $\mathcal{U}$ has a function from $A$ associated with it. Since $A$ is groupable we may partition it as $A=\bigcup_{n<\infty} B_{n}$, where each $B_{n}$ is finite, any two $B_{n}$ 's are disjoint from each other, and each neighborhood of 0 meets all but finitely many of the $B_{n}$ 's.

Define the strategy $\sigma$ for ONE in the game $\mathrm{G}_{\text {fin }}(\Omega, \Omega)$ on $X$ as follows: $\sigma(\emptyset)=\mathcal{U}$. Should TWO now respond with the finite set $T_{1} \subset \sigma(\emptyset)$, ONE first finds a finite set of functions $f_{U, F}, U \in T_{1}$ in $A$, and then chooses the least $n_{1}$ such that this set of functions are among the ones in $C_{1}=\bigcup_{j \leq n_{1}} B_{j}$. Then ONE plays $\sigma\left(T_{1}\right)$ to be all sets in $\mathcal{U} \backslash T_{1}$ not associated with any of the functions in $C_{1}$. If TWO now chooses the finite set $T_{2} \subset \sigma\left(T_{1}\right)$, then ONE first finds a finite set of functions in $A \backslash C_{1}$ of the form $f_{U, F}, U \in T_{2}$, and then chooses the least $n_{2}>n_{1}$ such that this set of functions are among the functions in $C_{2}=\bigcup_{n_{1}<j \leq n_{2}} B_{j}$; next ONE plays $\sigma\left(T_{1}, T_{2}\right)$ to be the set of $U \in \mathcal{U} \backslash\left(T_{1} \cup T_{2}\right)$ that are not associated with any of the functions in $C_{1} \cup C_{2}$, and so on.

Since $\sigma$ is not a winning strategy for ONE, consider a $\sigma$-play

$$
\sigma(\emptyset), T_{1}, \sigma\left(T_{1}\right), T_{2}, \sigma\left(T_{1}, T_{2}\right), T_{3}, \ldots
$$

which was won by TWO, together with the corresponding finite subsets $C_{1}, C_{2}, \ldots$ of $A$. Now expand $T_{1}$ to a larger finite set by including for each function in $C_{1}$ a corresponding set from $\mathcal{U}$ into $T_{1}$. Notice that this does not require moving an element of a later $T_{n}$ to $T_{1}$. Similarly, for each $n$ expand, if necessary, $T_{n}$ to include from $\mathcal{U}$ a set corresponding to each function in $C_{n}$. Again, this requires no reassignment of sets already in some $T_{j}$ to $T_{n}$. The resulting sequence of $T_{n}$ 's are finite and disjoint. We claim that $\bigcup_{n<\infty} T_{n}$ is groupable. For let $F$ be a finite subset of $X$, and consider the neighborhood 
$W=W(0, F, 1)$ of 0 . For all but finitely many $n$ we have $C_{n} \cap W \neq \emptyset$. Choose for any such $n$ an $f_{U, G} \in C_{n} \cap W$, where now $U \in T_{n}$. Then, as $f_{U, G}[F] \subset(-1,1)$, and as $f_{U, G}[X \backslash U]=\{1\}$, it follows that $F \subset U$. Thus, for all but finitely many $n$ there are $U \in T_{n}$ with $F \subset T_{n}$. Since the $T_{n}$ 's are pairwise disjoint, the set $\bigcup_{n<\infty} T_{n}$ is a groupable $\omega$-cover of $X$. Thus, $\mathcal{U}$ contains a groupable $\omega$-cover, and as it is countable, it is itself groupable.

REMARKs. 1 . If a space $X$ has property $\mathrm{S}_{\text {fin }}\left(\Omega_{x}, \Omega_{x}^{\mathrm{gp}}\right)$, then $X$ has countable fan tightness at $x$. But the converse is not true. Recall that a set of real numbers is a Lusin set if it is uncountable and its intersection with every first category set is countable. Lusin proved that under $\mathrm{CH}$ there exists a Lusin set. In [8] it was shown that $\mathrm{CH}$ implies the existence of a Lusin set $L$ all of whose finite powers have Rothberger's property. By a theorem of Sakai [18], $C_{p}(L)$ has countable strong fan tightness and thus countable fan tightness. But by a result of Hurewicz no Lusin set has the Hurewicz property. By Theorem 21, $\mathrm{C}_{\mathrm{p}}(L)$ does not have property $\mathrm{S}_{\text {fin }}\left(\Omega_{0}, \Omega_{0}^{\mathrm{gp}}\right)$.

2. By Theorem 21 and a result from [23] if $X$ is a perfectly normal space and $\mathrm{C}_{\mathrm{p}}(X)$ has property $\mathrm{S}_{\mathrm{fin}}\left(\Omega_{0}, \Omega_{0}^{\mathrm{gp}}\right)$, then $\mathrm{C}_{\mathrm{p}}(X)$ also has the monotonic sequence selection property. The converse need not be true. A Sierpinski set is an uncountable set of real numbers whose intersection with every set of Lebesgue measure zero is countable. Sierpiński proved that under $\mathrm{CH}$ there is a Sierpiński set. Let $S$ be the (special) Sierpiński set constructed under $\mathrm{CH}$ in [8]. Then $\mathrm{C}_{\mathrm{p}}(S)$ has the sequence selection property (and thus the monotonic sequence selection property) but does not have property $\mathrm{S}_{\text {fin }}\left(\Omega_{0}, \Omega_{0}^{\mathrm{gp}}\right)$, because it does not have countable fan tightness (see [23]).

According to [2] spaces $X$ and $Y$ are t-equivalent if the spaces $\mathrm{C}_{\mathrm{p}}(X)$ and $\mathrm{C}_{p}(Y)$ are homeomorphic.

Corollary 22. If $X$ and $Y$ are t-equivalent and if all finite powers of $X$ have the Hurewicz property, then all finite powers of $Y$ have the Hurewicz property.

If all finite powers of $X$ have the Hurewicz property, then all finite powers of $X \times \mathbb{N}$ have the Hurewicz property. Since $C_{p}(X \times \mathbb{N})$ is homeomorphic to $\mathrm{C}_{\mathrm{p}}(X)^{\omega}$, we have:

Corollary 23. If $\mathrm{C}_{\mathrm{p}}(X)$ has property $\mathrm{S}_{\mathrm{fin}}\left(\Omega_{0}, \Omega_{0}^{\mathrm{gp}}\right)$, then so does $\mathrm{C}_{\mathrm{p}}(X)^{\omega}$.

By Theorem 21 and a result from [7] we also have

Corollary 24. The minimal cardinality of a set $X$ of real numbers such that $\mathrm{C}_{\mathrm{p}}(X)$ does not have $\mathrm{S}_{\mathrm{fin}}\left(\Omega_{0}, \Omega_{0}^{\mathrm{gp}}\right)$ is $\mathfrak{b}$. 


\section{Countable strong fan tightness and the Reznichenko prop-} erty in $C_{p}(X)$. The main result in $[9]$ is:

TheOREM 25. For a Tikhonov space $X$ the following are equivalent.

(1) Each finite power of $X$ has both the Hurewicz property and $\mathrm{S}_{1}(\mathcal{O}, \mathcal{O})$.

(2) $\mathrm{C}_{\mathrm{p}}(X)$ has countable strong fan tightness as well as Reznichenko's property.

Using the techniques of this paper that result can be extended to the following:

Theorem 26. For a Tikhonov space $X$ the following are equivalent:

(1) $X$ has property $\mathrm{S}_{1}\left(\Omega, \Omega^{\mathrm{gp}}\right)$.

(2) $\mathrm{C}_{\mathrm{p}}(X)$ has property $\mathrm{S}_{1}\left(\Omega_{0}, \Omega_{0}^{\mathrm{gp}}\right)$.

(3) ONE has no winning strategy in the game $\mathrm{G}_{1}\left(\Omega_{0}, \Omega_{0}^{\mathrm{gp}}\right)$.

(4) For each $n$ and $k, \mathrm{C}_{\mathrm{p}}(X)$ satisfies $\Omega_{0} \rightarrow\left(\Omega_{0}^{\mathrm{gp}}\right)_{k}^{n}$.

\section{References}

[1] A. V. Arkhangel'skiŭ, Hurewicz spaces, analytic sets and fan tightness of function spaces, Soviet Math. Dokl. 33 (1986), 396-399.

[2] - Topological Function Spaces, Kluwer, 1992.

[3] J. E. Baumgartner, A. D. Taylor, Partition theorems and ultrafilters, Trans. Amer. Math. Soc. 241 (1978), 283-309.

[4] F. Galvin, Indeterminacy of point-open games, Bull. Acad. Polon. Sci. Sér. Sci. Math. Astronom. Phys. 26 (1978), 445-448.

[5] J. Gerlits and Zs. Nagy, Some properties of $C(X)$, I, Topology Appl. 14 (1982), $151-161$.

[6] W. Hurewicz, Über eine Verallgemeinerung des Borelschen Theorems, Math. Z. 24 (1925), 401-421.

[7] - Über Folgen stetiger Funktionen, Fund. Math. 9 (1927), 193-204.

[8] W. Just, A. W. Miller, M. Scheepers and P. J. Szeptycki, The combinatorics of open covers II, Topology Appl. 73 (1996), 241-266.

[9] Lj. Kočinac and M. Scheepers, Function spaces and a property of Reznichenko, Topology Appl. 123 (2002), 135-143.

[10] C. Laflamme and M. Scheepers, Combinatorial properties of filters and open covers for sets of real numbers, J. Symbolic Logic, to appear.

[11] V. I. Malykhin and G. Tironi, Weakly Fréchet-Urysohn and Pytkeev spaces, Topology Appl. 104 (2000), 181-190.

[12] K. Menger, Einige Überdeckungssätze der Punktmengenlehre, Sitzungsber. Wien Abt. 2a Math. Astronom. Phys. Meteorol. Mech. 133 (1924), 421-444.

[13] A. Nowik, M. Scheepers and T. Weiss, The algebraic sum of sets of real numbers with strong measure zero sets, J. Symbolic Logic 63 (1998), 301-324.

[14] J. Pawlikowski, Undetermined sets of point-open games, Fund. Math 144 (1994), 279-285.

[15] E. G. Pytkeev, On maximally resolvable spaces, Trudy Mat. Inst. Steklov. 154 (1983), 209-213 (in Russian); English translation: Proc. Steklov Inst. Math. 4 (1984), 225-230. 
[16] F. P. Ramsey, On a problem of formal logic, Proc. London Math. Soc. 30 (1930), 264-286.

[17] F. Rothberger, Eine Verschärfung der Eigenschaft C, Fund. Math. 30 (1938), 50-55.

[18] M. Sakai, Property $C^{\prime \prime}$ and function spaces, Proc. Amer. Math. Soc. 104 (1988), 917-919.

[19] M. Scheepers, Combinatorics of open covers I: Ramsey theory, Topology Appl. 69 (1996), 31-62.

[20] -, Combinatorics of open covers (III): games, $C_{\mathrm{p}}(X)$, Fund. Math. 152 (1997), 231-254.

[21] —, The least cardinal for which the Baire category theorem fails, Proc. Amer. Math. Soc. 125 (1997), 579-585.

[22] - Combinatorics of open covers ( $V)$ : Pixley Roy spaces of sets of reals, and $\omega$ covers, Topology Appl. 102 (2000), 13-31.

[23] - A sequential property of $C_{p}(X)$ and a covering property of Hurewicz, Proc. Amer. Math. Soc. 125 (1997), 2789-2795.

[24] -, Open covers and partition relations, Proc. Amer. Math. Soc. 127 (1999), 577-581.

[25] R. Telgársky, On games of Topsøe, Math. Scand. 54 (1984), 170-176.

Department of Mathematics

Faculty of Sciences

University of Niš

18000 Niš, Yugoslavia

E-mail: lkocinac@ptt.yu
Department of Mathematics Boise State University Boise, ID 83725, U.S.A. E-mail: marion@diamond.boisestate.edu

Received 20 March 2001; in revised form 8 April 2003 and 9 October 2003 